\title{
FUNCTIONS REPRESENTABLE AS DIFFERENCES OF SUBHARMONIC FUNCTIONS
}

\author{
BY \\ MAYNARD G. ARSOVE( $\left.{ }^{1}\right)$
}

1. Introduction. In what follows we investigate the behavior of functions of the form $u-v$, where $u$ and $v$ are subharmonic on a common open set of $n$-dimensional Euclidean space $\varepsilon^{n}$. Functions of this type will be called " $\delta$-subharmonic." A study of these functions has as its motivation the algebraic completion of the class of all subharmonic functions on a given open set, and we note also that all potentials and all sufficiently regular functions are $\delta$-subharmonic.

While certain isolated problems concerning $\delta$-subharmonic functions have been examined by Brelot, Privaloff, and others, no systematic treatment of the properties of $\delta$-subharmonic functions is available. We begin, therefore, by developing a general theory of such functions, outlining their basic properties and illustrating by counterexamples the main points of divergence between this theory and the theory of subharmonic functions.

As it turns out, not only does the extension from subharmonic to $\delta$-subharmonic functions result in closure as an additive group, but it also results in lattice closure: the upper and lower envelopes of any pair of $\delta$-subharmonic functions are themselves $\delta$-subharmonic. Moreover, certain further restrictions on the functions give rise to closure under multiplication. One would of course expect the gain in closure properties to be counterbalanced by a loss of some of the important properties of subharmonic functions, and this is indeed the case. Neither of the main convergence theorems for subharmonic functions (that for decreasing sequences and that for uniformly convergent sequences) remains valid for $\delta$-subharmonic functions. However, the loss is not quite a catastrophe, since by imposing supplementary conditions we manage to retrieve a useful convergence theorem in the $\delta$-subharmonic case.

The general theory of $\delta$-subharmonic functions enables us to introduce a characteristic function similar to that of Nevanlinna [1], but differing in an essential way from the characteristic function defined by Privaloff [1]. Applications of our characteristic function are immediate and yield, for example,

Presented to the International Congress of Mathematicians, September 4, 1950; received by the editors March 21, 1952.

(1) This paper is based on a portion of the author's thesis, submitted in partial fulfillment of the requirements for the degree of Doctor of Philosophy at Brown University. The author wishes to express his appreciation to Professor M. H. Heins, under whose direction the thesis was written, for numerous suggestions and criticisms, and to Professor M. Brelot of the University of Grenoble for a critical reading of the manuscript. Acknowledgment is due also to the referee for helpful comments and suggestions. 
$\delta$-subharmonic analogues of the classical theorems on isolated singularities of subharmonic functions. Further applications to the theory of entire $\delta$-subharmonic functions and the associated theory of functions of potential type will appear in a subsequent paper.

In view of the intrinsic connection between subharmonic functions and the theory of functions of a complex variable we shall center our attention primarily on the space $\mathcal{E}^{2}$. Notational conventions will be explained as introduced, and a glossary of these conventions is appended.

2. Fundamental definitions. We now formulate in a precise way the definition of $\delta$-subharmonicity and several allied concepts.

Definition 1. A function $w$ will be said to be $\delta$-subharmonic on an open subset $\Omega$ of $\varepsilon^{n}$ provided there exist functions $u$ and $v$ subharmonic on $\Omega$ such that (1) $w$ has as domain the set $D$ of points of $\Omega$ at which either $u$ or $v$ is finite, and (2) $w=u-v$ holds in the extended sense on $D$.

Although $w$ need not be defined throughout $\Omega$, the set of points at which $w$ fails to be defined finitely is clearly of capacity zero and therefore of Lebesgue measure zero.

A function which coincides almost everywhere with a function $\delta$-subharmonic on $\Omega$ will be called almost $\delta$-subharmonic on $\Omega$. For a useful further variant of $\delta$-subharmonicity we need the notion of "quasi everywhere" (Brelot [4]), which we recall briefly. A set $P\left(\subset \mathcal{E}^{n}\right)$ is said to be polar if there exists a subharmonic function on $\varepsilon^{n}$ assuming the value $-\infty$ at all points of $P$. Then by quasi everywhere on a set $E\left(\subset \varepsilon^{n}\right)$ we mean "except on a polar subset of $E$." With this in hand we say that a function is quasi $\delta$-subharmonic on $\Omega$ provided it coincides quasi everywhere with a function $\delta$-subharmonic on $\Omega$.

It should be remarked that an alternative theory of $\delta$-subharmonic functions, which differs from that presented here mainly in the domains of the functions involved, can be obtained by using in place of $\delta$-subharmonic functions, functions which are quasi $\delta$-subharmonic on $\Omega$ and which coincide on their domains with functions $\delta$-subharmonic on $\Omega$. These are the functions "locally potential" of Brelot [2].

The 1 -dimensional case of $\delta$-subharmonicity has a special significance because on $\mathcal{E}^{1}$ subharmonicity is identical with convexity. Results here are not only of interest in themselves, but they are also simpler to obtain and lead by analogy to general properties of $\delta$-subharmonic functions.

Definition 2. A function $f$ representable on an open subset $G$ of $\varepsilon^{1}$ as the difference of two convex functions will be called $\delta$-convex on $G$.

Since a $\delta$-subharmonic function can be represented as a difference of subharmonic functions in an infinite variety of ways, it is convenient to introduce the following terminology.

Definition 3. By a representation of a function $w \delta$-subharmonic (almost $\delta$-subharmonic, quasi $\delta$-subharmonic) on an open set $\Omega$ we mean an ordered 
pair $(u, v)$ of functions subharmonic on $\Omega$ such that $w=u-v$ holds (almost everywhere, quasi everywhere) on the domain of $w$.

3 . The local character of $\delta$-subharmonicity. Canonical representations. Completion. The question naturally arises as to whether $\delta$-subharmonicity, in spite of its global definition, is not also a local property. As Brelot has noted (Brelot [6]), the answer here is in the affirmative. We give a proof of this fact and indicate some of its consequences.

It is clear that the Riesz decomposition of a subharmonic function on a region $\Omega$ gives rise to a negative mass distribution $\left({ }^{2}\right)$ on $\Omega$. The converse has been established by Brelot [3]. Since this result plays a key role in the theory, we sketch a proof (slightly different from that of Brelot) for the case of $\varepsilon^{2}$.

THEOREM 1. If $m$ is a negative mass distribution on a region $\Omega$, then there exists a subharmonic function $u$ on $\Omega$ having $m$ as its mass distribution $\left(^{3}\right)$.

Proof. We employ a familiar exhaustion of $\Omega$ by a sequence $\left\{\Omega_{n}\right\}$ of bounded subregions such that (1) for all $n, \bar{\Omega}_{n} \subset \Omega_{n+1}$, (2) each $\Omega_{n}$ has its boundary composed of a finite number of disjoint Jordan curves, and (3) for every $n$ each Jordan curve forming the boundary of $\Omega_{n}$, except possibly that forming the outer boundary of $\Omega_{n}$, encloses at least one boundary point of $\Omega$. We shall denote $\bar{\Omega}_{n+1}-\bar{\Omega}_{n}$ by $A_{n}$ and define $u_{0}$ as the potential of $m$ on $\bar{\Omega}_{1}$ and $u_{n}$ for $n \geqq 1$ as the potential of $m$ on $A_{n}$.

The main problem is to prove that $u_{n+1}$ can be uniformly approximated on $\bar{\Omega}_{n}$ by functions harmonic on $\Omega$. For this we fix $n$ and denote the bounding Jordan curves of $\Omega_{n+1}$ by $\gamma_{0}, \gamma_{1}, \cdots, \gamma_{p}$. Taking $\gamma_{0}$ as the outer boundary, we know that each $\gamma_{k}$ with positive index encloses a boundary point $t_{k}$ of $\Omega$. Further, we see that $A_{n+1}$ consists of $p+1$ component half-open regions $\Theta_{k}$ corresponding to the curves $\gamma_{k}$. We denote by $v_{k}$ the potential of $m$ on $\Theta_{k}$.

It follows from a corollary of a theorem of Runge (Walsh [1]) that any function harmonic on a simply-connected region can be uniformly approximated on compact subsets of that region by harmonic polynomials. Hence, $v_{0}$ can be uniformly approximated on $\bar{\Omega}_{n}$ by harmonic polynomials. For positive $k$ the function $v_{k}(z)-m\left(\Theta_{k}\right) \log \left|z-t_{k}\right|$ is harmonic outside of $\gamma_{k}$, at infinity as well as at all finite points. Thus, by first performing an inversion about $t_{k}$ and then invoking the corollary of Runge's theorem, we see that $v_{k}$ can be uniformly approximated on $\bar{\Omega}_{n}$ by functions harmonic everywhere

(2) We employ the terminology "mass distribution" in its present measure-theoretic sense, corresponding to the terminology "generalized mass distribution" as used by Rad6 [1]: mass distribution on $\Omega=$ Radon measure on $\Omega=$ signed Borel measure on $\Omega$. A mass distribution may thus fail to admit a potential.

(3) This theorem finds its natural background in the Mittag-Leffler, Weierstrass theory for functions of a complex variable. It can, in fact, be proved for $\Omega=\boldsymbol{\varepsilon}^{2}$ (or for $\Omega=$ any circular subregion of $\varepsilon^{2}$ ) by use of an integral analogue of the Weierstrass infinite product representation for entire functions. 
except at $t_{k}$. This proves that $u_{n+1}$ can be uniformly approximated on $\bar{\Omega}_{n}$ by functions harmonic on $\Omega$.

If for every positive integer $n$ we choose $U_{n+1}$ harmonic on $\Omega$ such that

$$
\left|u_{n+1}-U_{n+1}\right| \leqq 1 / 2^{n} \text { on } \bar{\Omega}_{n}
$$

and put $U_{0} \equiv U_{1} \equiv 0$, then the series $\sum_{n=0}^{\infty}\left(u_{n}-U_{n}\right)$ defines on $\Omega$ a subharmonic function having $m$ as its mass distribution.

For the transition to $\delta$-subharmonic functions we have use for

Definition 4. A function $w \delta$-subharmonic on an open set $\Omega$ and having domain $D$ will be said to be complete provided every $\delta$-subharmonic function on $\Omega$ coinciding with $w$ on $D$ has $D$ as its domain.

In other words, a $\delta$-subharmonic function on $\Omega$ is complete if and only if it does not admit an extension of definition on $\Omega$ preserving $\delta$-subharmonicity.

THEOREM 2. To each function $\delta$-subharmonic (almost $\delta$-subharmonic, quasi $\delta$-subharmonic) on an open set $\Omega$ there corresponds, in a sense made clear by the Riesz decomposition theorem, a unique mass distribution on $\Omega$. Conversely, given a mass distribution $m$ on $\Omega$, there exists a complete $\delta$-subharmonic function on $\Omega$ having $m$ as its mass distribution.

Proof. The first part of the theorem is immediate. For the converse we split $m$ into its positive and negative parts. Theorem 1 yields subharmonic functions $u$ and $v$ corresponding to these parts, such that $(u, v)$ is a representation of a $\delta$-subharmonic function $w$ having the mass distribution $m$. The completeness of $w$ is then established by a local application of the Riesz decomposition theorem.

That local $\delta$-subharmonicity implies $\delta$-subharmonicity (in the global sense of definition 1) follows directly from this.

THEOREM 3. If $w$ is a function $\delta$-subharmonic (almost $\delta$-subharmonic, quasi $\delta$-subharmonic) in the neighborhood of each point of an open set $\Omega$, then $w$ is $\delta$-subharmonic (almost $\delta$-subharmonic, quasi $\delta$-subharmonic) on $\Omega$.

Proof. Local $\delta$-subharmonicity engenders local mass distributions, which (by the uniqueness of the Riesz mass) agree on overlapping sets and hence define a mass distribution on $\Omega$. It is readily seen that any complete $\delta$-subharmonic function $W$ having this mass distribution determines a harmonic function $h$ on $\Omega$ such that $w=W+h$ holds on the domain of $w$.

To conclude that $w$ is $\delta$-subharmonic, we need only exhibit a subharmonic function assuming the value $-\infty$ at those and only those points of $\Omega$ at which $w$ is undefined. It is clear that $\Omega$ can be expressed as a countable union of neighborhoods on each of which $w$ is $\delta$-subharmonic, and by ignoring overlaps we can replace these neighborhoods by a countable family of disjoint Borel sets. On each of these sets we consider the negative mass distribution corresponding to the upper envelope of $u$ and $v$, where $(u, v)$ is a local 
representation of $w$, and in this fashion obtain a negative mass distribution on $\Omega$ for which any of the corresponding subharmonic functions has the required distribution of infinities.

Among all possible representations of a given $\delta$-subharmonic function there is one of a particularly simple nature and determined to within a certain degree of uniqueness. This representation, which will be seen to play an essential part in our formulation of a characteristic function, is termed the "canonical representation" of the given function.

Definition 5. Let $w$ be a $\delta$-subharmonic (almost $\delta$-subharmonic, quasi $\delta$-subharmonic) function, whose mass distribution has positive part $p$ and negative part $n$. A representation $(u, v)$ of $w$ will be called canonical provided the mass distribution for $u$ is $-n$ and that for $v$ is $-p$.

By separating the mass distribution for a given $\delta$-subharmonic function into its positive and negative parts and applying Theorem 1 , we readily obtain

THEOREM 4. Every $\delta$-subharmonic (almost $\delta$-subharmonic, quasi $\delta$-subharmonic) function $w$ admits a canonical representation $(u, v)$, the functions $u$ and $v$ being unique to within a common additive harmonic function. Moreover, the $\delta$-subharmonic function $u-v$ defined at all points for which $u$ and $v$ are not both $-\infty$ is complete.

In particular, this tells us that every function $\delta$-subharmonic on an open set $\Omega$ admits a unique complete extension on $\Omega$.

Although canonical representations have been defined in terms of mass distributions, it is of interest to note that they can be characterized by extremal properties in such a way as to avoid all reference to mass distributions.

THEOREM 5. The canonical representations of a function $w \delta$-subharmonic (almost $\delta$-subharmonic, quasi $\delta$-subharmonic) on an open set $\Omega$ are characterized by the following property of minimal subharmonicity. A representation $(u, v)$ of $w$ is canonical if and only if to each representation $(U, V)$ of $w$ there corresponds a function $S$ subharmonic on $\Omega$ such that

$$
U=u+S \text { and } V=v+S
$$

hold on the domains of $U$ and $V$, respectively.

The proof depends simply on the minimal nature of the positive and negative parts of the mass distribution for $w$.

4. $\delta$-convex functions. Functions of this sort were considered by F. Riesz [1] as early as 1911 and have been studied more recently by Brelot, Zygmund, and others. Their theory is, of course, much easier than that of $\delta$-subharmonic functions in higher dimensional spaces, in view of the wealth of analytical machinery available for the real line. 
However, we shall not be concerned here with collecting a vast amount of data on $\delta$-convex functions. Instead, we state a few well known elementary results, which will serve to orient our thinking as to the possible behavior of $\delta$-subharmonic functions in general.

As shown by Brelot $\left(^{4}\right)$ the choice of $|x-t|$ for the potential kernel in $\varepsilon_{1}$ yields results for convex functions, hitherto established only for subharmonic functions in $\mathcal{E}^{n}$ for $n \geqq 2$. Consequently, for $\delta$-convex functions we have at our disposal the local properties discussed in $\S 3(5)$. Certain other properties, notably continuity, are peculiar to the 1-dimensional case.

These special properties of $\delta$-convex functions lead to very precise results, which have only approximate analogues in higher dimensions.

Theorem 6. A function $f$ is $\delta$-convex on an open set if and only if it can be expressed on every compact subinterval as the indefinite integral of a function of bounded variation on that subinterval.

Thus, the continuity of a $\delta$-convex function is absolute on compact subintervals. A further criterion for $\delta$-convexity has been given by L. Schwartz in terms of the theory of distributions (Schwartz [1, p. 54]).

THEOREM 7. A function $f$ is $\delta$-convex on an open set if and only if its second derivative in the sense of the theory of distributions is a measure.

However, perhaps the most useful characterization of $\delta$-convexity is the following one, in which only the first derivative appears.

THEOREM 8. A function $f$ on an open set $G$ is $\delta$-convex if and only if (1) $f$ is continuous and (2) $f^{\prime}$ exists finitely at all except perhaps a countable set of points of $G$ and is locally of bounded variation on the subset on which it exists finitely.

For the sake of comparison with corresponding sufficient conditions for $\delta$-subharmonicity we state the evident

Corollary 8.1. Any of the following conditions ensures $\delta$-convexity of $a$ function $f$ on an open set $G$ :

(1) the graph of $f$ is a polygonal arc whose vertices do not have a limit point over $G$;

(2) $f^{\prime \prime}$ exists and is locally bounded on $G$;

(3) $f^{\prime}$ exists and satisfies a locally uniform Lipschitz condition on $G$.

Theorem 8 permits us also to describe concisely the family of all $\delta$-convex functions on a given open set.

(4) This material appears in lectures, as yet unpublished.

(5) An alternative way of arriving at these properties is to use the corresponding properties for the plane together with the following two principles: (i) if $f$ is convex, then $f(\log |z|)$, $|z|>0$, is subharmonic, and (ii) if $u$ is subharmonic, then its circumferential mean is convex in $\log r$. 
Corollary 8.2. The family of all functions $\delta$-convex on an open set $G$ forms an algebra over the real field with respect to the ordinary operations of addition and multiplication of functions and of multiplication of functions by reals $\left(^{6}\right)$.

5. Criteria for $\delta$-subharmonicity and almost $\delta$-subharmonicity. The Wiener variation. Although the necessary and sufficient conditions of the preceding section cannot be paralleled exactly for higher dimensions, it is possible to give a simple characterization of $\delta$-subharmonicity in terms of the Riesz decomposition. However, the $\delta$-convexity criterion which admits the closest analogue is that given in terms of distributions (Theorem 7), but its counterpart is a criterion for almost $\delta$-subharmonicity, rather than for $\delta$-subharmonicity.

Local application of the Riesz decomposition theorem yields

TheOREM 9. A necessary and sufficient condition that a function w be a complete $\delta$-subharmonic function on an open set $\Omega$ is that $w$ be expressible locally on $\Omega$ as a potential plus a harmonic function.

Deny has shown (Deny [1]) that given any polar $G_{\delta}$ set $E$ in $\mathcal{E}^{n}(n \geqq 2)$, there exists a positive mass distribution whose potential is infinite throughout $E$ and finite at all other points of $\varepsilon^{n}$. Conversely, it is clear that the set of points at which a subharmonic function assumes the value $-\infty$ is a polar $G_{\delta}$. Since the set of points at which a $\delta$-subharmonic function with representation $(u, v)$ is undefined is exactly the set on which the upper envelope of $u$ and $v$ assumes the value $-\infty$, we have

Corollary 9.1. A necessary and sufficient condition for a function w to be $\delta$-subharmonic on an open set $\Omega$ is that there exist a polar $G_{\delta}$ set $E$ such that $w$ has domain $\Omega-E$ and is expressible in the neighborhood of each point of $\Omega-E$ as a potential plus a harmonic function.

One might at first hope to be able to characterize $\delta$-subharmonicity also in terms of the behavior at each point of the circumferential or areal means in a manner similar to that for subharmonicity, but the following trivial example serves to dispel this hope.

EXAmple 1. Let $w$ be defined on $\varepsilon^{2}$ as 1 on the left half-plane $(x<0), 0$ on the $y$-axis, and -1 on the right half-plane $(x>0)$. Then $w$ is the difference of two upper semi-continuous functions, and the circumferential and areal means taken over sufficiently small circles about any point $z$ have the constant value $w(z)$. However, $w$ is not even almost $\delta$-subharmonic, in view of a theorem of Evans (Radó [1, p. 45]) which forces a $\delta$-subharmonic function $w(x, y)$ to be absolutely continuous in $x$ for almost all $y$.

For almost $\delta$-subharmonicity a very concise criterion is available in terms of the theory of distributions of L. Schwartz [2].

(6) An algebraic proof of this fact appears in Theorem 25. 
THEOREM 10. Let w be a function defined almost everywhere on an open set $\Omega$ and summable on compact subsets of $\Omega$. A necessary and sufficient condition for $w$ to be almost $\delta$-subharmonic is that $\Delta w$ be a measure, where $\Delta$ denotes the generalized Laplacian of Schwartz.

Shorn of the elegant distribution-theoretic formulation given by Schwartz, this result seems to go back, in essence, at least to Zaremba [1]. The classical condition, which we state below, is in fact somewhat more precise than that in terms of distributions. Since it appears rather difficult to trace down a simple derivation of this condition, we shall outline briefly its proof.

As a notational convenience, we restrict ourselves to the plane $\mathcal{E}^{2}$ for the remainder of this section, but the results generalize immediately to any $\varepsilon^{n}$. Lebesgue plane measure will be denoted by $a$.

THEOREM 11. Let $w$ be a function defined almost everywhere on an open set $\Omega$ and summable on compact subsets of $\Omega$. A necessary and sufficient condition for $w$ to be almost $\delta$-subharmonic on $\Omega$ is that to each compact subset $K$ of $\Omega$ there correspond a constant $\gamma(K)$ such that for all functions $f \in C^{2}(\Omega)$ with support $K$

$$
\left|\int_{\Omega} w \Delta f d a\right| \leqq \gamma(K) \max |f| .
$$

Furthermore, under these conditions

$$
(-1 / 2 \pi) \int_{\Omega} w \Delta f d a=\int_{\Omega} f d m,
$$

where $m$ is the mass distribution for $w$.

Proof. (Necessity). The subclass of $C^{2}(\Omega)$ consisting of those functions supported by compact subsets of $\Omega$ will be designated by $\mathcal{C}^{2}$. Classical potential theory then yields the result that every $f \in \mathcal{C}^{2}$ is the potential of the mass distribution having density $-\Delta f / 2 \pi$. For a given $K$ we pick $\Omega^{*}$ as a bounded open set containing $K$ and having closure in $\Omega$. Then almost everywhere on $\Omega^{*}$ we have $w=w^{*}+h^{*}$, where $w^{*}$ is the potential of $m$ on $\Omega^{*}$ and $h^{*}$ is harmonic on $\Omega^{*}$. From Green's theorem it is clear that

$$
\int_{\Omega^{*}} h^{*} \Delta f d a=0,
$$

and (5.2) follows from the reciprocity theorem (Fubini's theorem).

(Sufficiency). Under the uniform topology $\mathcal{C}^{2}$ is dense in the space $\mathcal{C}$ of all continuous functions on $\Omega$ supported by compact subsets. Hence, the linear functional $L$ defined on $\mathcal{C}^{2}$ by

$$
L(f)=(-1 / 2 \pi) \int_{\Omega} w \Delta f d a
$$


can be extended to $\mathcal{C}$, and (5.1) ensures that the resulting linear functional will be bounded. From the Riesz representation theorem for bounded linear functionals on $\mathcal{C}$ it follows that there exists a mass distribution $m$ on $\Omega$ such that (5.2) holds. Taking $W$ as any $\delta$-subharmonic function on $\Omega$ having mass distribution $m$ thereby yields

$$
\int_{\Omega}(w-W) \Delta f d a=0
$$

for all $f \in \mathcal{C}^{2}$, and this persists for $w-W$ replaced by its triple areal means. Since the latter are then harmonic (by Green's theorem) and converge in the mean to $w-W$, it follows that $w-W$ must be almost harmonic on $\Omega$.

In an article published at the request of F. Riesz, N. Wiener [1] has developed a representation for continuous linear functionals similar to that of the classical Riesz representation theorem. Wiener states as his aim a circumvention of dependence on the choice of axis system inherent in the Riesz representation, and he carries out the details with the use of the theory of Fourier integrals. We propose to derive the Wiener representation from that of Riesz and to correlate the two in terms of the theory of almost $\delta$-subharmonic functions.

By analogy between the finite difference quotient for a function $f$ of one variable and the Blaschke difference operator for a function $w$ of several variables, Wiener defines a "total variation" for the function $w$. However, it should be noted that this total variation is more appropriately a "variation in convexity," since the Blaschke difference operator corresponds to the second, rather than the first, derivative of $f$. We shall employ the terminology "Wiener variation" for this functional and show that local boundedness of the Wiener variation is equivalent to almost $\delta$-subharmonicity, a result due in part to Rosenbloom [1].

The circular neighborhood of radius $r$ and center $z$ will be denoted by $N_{r}(z)$, its closure by $S_{r}(z)$, and its boundary by $C_{r}(z)$. Then for $w$ an integrable function, $\mu_{r} w(z)$ will signify the integral mean of $w$ over $C_{r}(z)$, and $\alpha_{r} w(z)$ the integral mean over $S_{r}(z)$. In this notation the definition of the Blaschke difference operator $\Delta_{r}^{B}$ appears as

$$
\Delta_{r}^{B} w(z)=\left(4 / r^{2}\right)\left[\mu_{r} w(z)-w(z)\right] .
$$

The corresponding Privaloff difference operator $\Delta_{r}^{P}$ is defined by replacing $\mu_{r} w$ by $\alpha_{r} w$ and the factor $4 / r^{2}$ by $8 / r^{2}$.

Definition 6. Let $w$ be a measurable function defined finitely almost everywhere on an open set $\Omega$ and integrable on compact subsets of $\Omega$. By the Wiener variation of $w$ on a measurable subset $E$ of $\Omega$ we mean the quantity $\psi_{E}(w)$ defined in the extended sense as

$$
\psi_{E}(w)=\limsup _{r \rightarrow 0}(1 / 2 \pi) \int_{E_{r}}\left|\Delta_{r}^{B} w\right| d a,
$$


where $E_{r}$ denotes the set consisting of those points of $E$ whose distance from the boundary of $\Omega$ exceeds $r\left({ }^{7}\right)$.

It is obvious that $\psi_{E}$ has the usual elementary properties of a variation functional: (1) $\psi_{E}(w) \geqq 0, \quad(2) \psi_{E_{1}}(w) \leqq \psi_{E_{2}}(w)$ for $E_{1} \subset E_{2}$, (3) $\psi_{E}(c w)$ $=|c| \psi_{E}(w)$ for $c$ a real number, and (4) $\psi_{E}\left(w_{1}+w_{2}\right) \leqq \psi_{E}\left(w_{1}\right)+\psi_{E}\left(w_{2}\right)$.

The importance of the Wiener variation in potential theory was first recognized by Rosenbloom [1], who devised an elegant measure-theoretic summability technique for estimating the Wiener variation of potentials $\left({ }^{8}\right)$. Lemma 1 and Theorems 12 and 13 (which follow) are results of Rosenbloom, modified in an unessential way by insertion of the function $f$ in the inte$\operatorname{grands}\left({ }^{9}\right)$.

Lemma 1. Let $w$ be the potential of a mass distribution $m$, and let $f$ be a bounded Borel measurable function on $\mathcal{E}^{2}$. Further, let $e$ be a bounded Borel set, and let us denote by $M_{e}^{*}(f, z, t)$ the total mass on the neighborhood $N_{t}(z) d u e$ to the mass distribution of density $f$ on $e$ :

$$
M_{e}^{*}(f, z, t)=\int_{e \cap_{N t(z)}} f d a .
$$

If we set

$$
\delta_{e}(f, r, z)=\left(2 / \pi r^{2}\right) \int_{0}^{r}(1 / t) M_{e}^{*}(f, z, t) d t
$$

then

$$
\int_{\varepsilon^{2}} \delta_{e}(f, r, z) d m(z)=(-1 / 2 \pi) \int_{e} f \Delta_{r}^{B} w d a .
$$

Proof. An integration by parts yields

$$
\begin{aligned}
\int_{\mathcal{E}^{2}} \delta_{e}(f, r, z) d m(z) & =\left(2 / \pi r^{2}\right) \int_{\mathcal{E}^{2}} \int_{0}^{r} \log (r / t) d M_{e}^{*}(f, z, t) d m(z) \\
& =(-1 / 2 \pi) \int_{\mathcal{E}^{2}} \Delta_{r}^{B} w^{*} d m,
\end{aligned}
$$

where $w^{*}$ is the potential due to the mass distribution of density $f$ on $e$. The lemma follows by an application of Fubini's theorem.

Taking $e^{r}$ as the set of all points whose distance from $e$ is less than $r$ leads to on $E_{r}$.

(7) Note that $E_{r}$ is measurable and that $\Delta_{r}^{B}$ is defined almost everywhere and measurable

(8) The author wishes to express his gratitude to Professor P. C. Rosenbloom for the opportunity of reading his manuscript prior to its publication.

$\left({ }^{9}\right)$ As Rosenbloom has shown, Theorem 13 (with $f=1$ ) yields a concise proof of the uniqueness of the mass distribution producing a given potential. 


$$
(1 / 2 \pi) \int_{e}\left|\Delta_{r}^{B} w\right| d a \leqq \int_{e} \delta_{e}(1, r, z)|d m(z)| \leqq \int_{e^{r}}|d m| .
$$

There follows

THEOREM 12. Let $w$ be a function almost $\delta$-subharmonic on an open set $\Omega$. If we denote by $\phi_{e}(w)$ the total variation of the mass distribution for $w$ on any Borel subset $e$ of $\Omega$, then for all such sets $\psi_{e}(w) \leqq \phi_{i \cap \Omega}(w)$.

A further consequence of the lemma is

THEOREM 13. Let $w$ be the potential of a mass distribution $m$, and let $f$ be a bounded continuous function on $\mathcal{E}^{2}$. Then for all bounded Borel sets $e$ having no mass on their boundaries

$$
\lim _{r \rightarrow 0}(-1 / 2 \pi) \int_{0} f \Delta_{r}^{B} w d a=\int_{0} f d m .
$$

Proof. Employing the notation of the lemma and denoting by $\chi_{e}$ the characteristic function of $e$, we see from the continuity of $f$ that

$$
\lim _{r \rightarrow 0} \delta_{e}(f, r, z)=\chi_{e}(z) f(z)
$$

holds for all $z$ not on the boundary of $e$. It is clear also by the boundedness of $f$ that $\delta_{e}(f, r, z)$ is bounded. Hence,

$$
\begin{aligned}
\lim _{r \rightarrow 0}\left[(-1 / 2 \pi) \int_{e} f \Delta_{r}^{B} w d a-\int_{e} f d m\right] & \\
= & \lim _{r \rightarrow 0} \int_{\varepsilon^{2}}\left[\delta_{e}(f, r, z)-\chi_{e}(z) f(z)\right] d m(z)=0
\end{aligned}
$$

in view of the fact that $e$ has no mass on its boundary.

Theorem 13 can be obtained also by means of the theory of distributions. It suffices to prove that the mass distributions $m_{r}$ defined by

$$
m_{r}(e)=(-1 / 2 \pi) \int_{e} \Delta_{r}^{B} w d a
$$

converge weakly $\left({ }^{10}\right)$ to $m$, and for this we define $p_{r}$ as the mass distribution formed by distributing a unit mass uniformly on $C_{r}(0), r \geqq 0$. We consider the mass distribution

$$
T_{r}=\left(4 / r^{2}\right)\left(p_{r}-p_{0}\right)
$$

as a distribution of Schwartz and show that $T_{r}$ converges to the $\Delta$ of Schwartz. Here we take $\mathcal{F}$ as any family of infinitely differentiable functions $\phi$ whose

(10) See Definition 7 and subsequent remarks. 
supports lie in a fixed bounded set and having the property that $\mathcal{F}$, as well as each family formed by taking derivatives of any fixed order of the functions $\phi$, is bounded. Then a classical argument based on the Taylor expansion yields

$$
\lim _{r \rightarrow 0} T_{r}(\phi)=\phi_{x x}(0)+\phi_{y y}(0)
$$

uniformly for $\phi$ in $\mathcal{F}$. That is, $T_{r}$ tends to $\delta_{x x}+\delta_{y y}$ in the sense of distributions, where $\delta$ is the Dirac distribution. Denoting the operation of convolution by an asterisk and recalling the expression for the $\Delta$ of Schwartz as $\Delta w$ $=\left(\delta_{x x}+\delta_{y y}\right) * w$, we see that $T_{r} * w$ converges to $\Delta w=-2 \pi m$. Moreover, a direct computation verifies that

$$
\left(T_{r} * w\right)(\phi)=\int \phi \Delta_{r}^{B} w d a .
$$

Since the functions of the form $\phi$ constitute a total set in the sense of Banach [1], it follows that $m_{r}$ converges weakly to $m$.

We propose to show next that local finiteness of the Wiener variation implies almost $\delta$-subharmonicity.

THEOREM 14. Let $w$ be a function defined almost everywhere on an open set $\Omega$ and summable on compact subsets of $\Omega$. A necessary and sufficient condition for $w$ to be almost $\delta$-subharmonic on $\Omega$ is that $\psi_{K}(w)$ be finite for all compact subsets $K$ of $\Omega$.

Proof. The necessity is obvious from Theorem 12. For the sufficiency we take $f$ as any function in $C^{2}(\Omega)$ vanishing outside of a compact subset $K$. A simple computation yields

$$
\int_{\Omega} w \Delta_{r}^{B} f d a=\int_{\Omega} f \Delta_{r}^{B} w d a,
$$

from which there results

$$
\left|\int_{\Omega} w \Delta_{r}^{B} f d a\right| \leqq\left(\int_{K}\left|\Delta_{r}^{B} w\right| d a\right) \max |f| .
$$

By virtue of the hypotheses on $f, \Delta_{r}^{B} f$ converges boundedly to $\Delta f$, so that in the limit as $r \rightarrow 0$ we obtain

$$
\left|\int_{\Omega} w \Delta f d a\right| \leqq 2 \pi \psi_{K}(w) \max |f| .
$$

An application of Theorem 11 completes the proof.

Turning now to the Wiener representation theorem, we take $\Omega$ as any open set and $L$ as a bounded linear functional defined on the class $\mathcal{C}$ of all 
continuous functions on $\Omega$ having compact supports. The Riesz representation theorem asserts the existence of a unique mass distribution $m$ on $\Omega$ such that $L(f)=\int_{\Omega} f d m$ holds for all $f \in \mathcal{C}$. If $w$ is any $\delta$-subharmonic function on $\Omega$ having mass distribution $m$, then by Theorem 13 we obtain

$$
L(f)=\lim _{r \rightarrow 0}(-1 / 2 \pi) \int_{\Omega} f \Delta_{r}^{B} w d a .
$$

Thus, the Wiener representation theorem (stated below) is a consequence of the classical Riesz theorem.

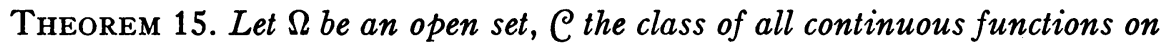
$\Omega$ having compact supports, and $L$ a bounded linear functional on $C$. Then there exists a function $w$ on $\Omega$, having finite Wiener variation on compact subsets and determined uniquely almost everywhere to within an additive harmonic function, such that

$$
L(f)=\lim _{r \rightarrow 0}(-1 / 2 \pi) \int_{\Omega} f \Delta_{r}^{B} w d a
$$

for all $f \in C$.

In addition to the criteria for $\delta$-subharmonicity and almost $\delta$-subharmonicity, a criterion can be given for quasi $\delta$-subharmonicity in terms of almost $\delta$-subharmonicity and the behavior of the circumferential or areal means.

THEOREM 16. A necessary and sufficient condition that a function w defined quasi everywhere on an open set $\Omega$ be quasi $\delta$-subharmonic on $\Omega$ is that $w$ be almost $\delta$-subharmonic and satisfy

$$
w=\lim _{r \rightarrow 0} \mu_{r} w \quad\left[\text { or } w=\lim _{r \rightarrow 0} \alpha_{r} w\right]
$$

quasi everywhere on $\Omega$.

Proof. Since $w$ is almost $\delta$-subharmonic, it coincides almost everywhere with a function $W \delta$-subharmonic on $\Omega$. The theorem follows from the fact that the circumferential and areal means of $W$ tend to $W$ quasi everywhere as $r \rightarrow 0$.

6. Some sufficient conditions for $\delta$-subharmonicity. It goes without saying that the criterion for $\delta$-subharmonicity given in Corollary 9.1 is frequently difficult to apply. We therefore develop certain conditions sufficient to ensure $\delta$-subharmonicity, and which, in spite of their restrictive nature, have important applications. They yield, in fact, $\delta$-subharmonic functions bearing little superficial relation to potential theory.

Let us begin by trying to find analogues of the $\delta$-convexity conditions 
mentioned in Corollary 8.1 .

THEOREM 17. Any function on an open subset of $\varepsilon^{2}$ whose graph is a polyhedral surface (that is, a continuous piecewise planar surface) with faces locally finite in number is $\delta$-subharmonic.

Proof. In view of the harmonicity of linear functions it is only necessary to verify $\delta$-subharmonicity in the neighborhood of the edges and vertices. This is trivial for the edges, since on any neighborhood of an edge not containing a vertex the function is either the upper or the lower envelope of the two planes forming the faces, and is therefore either subharmonic or superharmonic. The reasoning is slightly more complicated for the vertices.

By inserting additional edges if necessary, we can assume that successive edges about a fixed vertex form angles less than a right angle. Taking $p, q$, and $r$ as three successive edges, we then see that the faces $p q$ and $q r$ lie either both above or both below (or on) the plane pr. The polyhedral surface formed by adjoining to faces $p q$ and $q r$ the appropriate portion of the plane $p r$ is then either $\max [p r, \min (p q, q r)]$ or $\min [p r, \max (p q, q r)]$. As is shown later in Theorem 22, both of these functions are $\delta$-subharmonic. Subtracting this new polyhedral surface from the given surface replaces $p q$ and $q r$ by faces which are horizontal.

Let us suppose now that $s$ and $t$ are edges taken in succession after $p, q, r$ and that (by virtue of the above transformation) $p, q$, and $r$ are horizontal. If $t^{\prime}$ is the horizontal ray through the vertex and lying in the same vertical plane as $t$, our previous argument shows that the polyhedral surface determined by $r, s, t^{\prime}$ represents a $\delta$-subharmonic function. Subtraction of this polyhedral surface replaces $r s$ by a horizontal face and at the same time leaves the faces $p q$ and $q r$ horizontal. An iteration of this process yields a surface identically constant, proving that the given function is the sum of a finite number of $\delta$-subharmonic functions in the neighborhood of the given vertex.

Although the above derivation has the merit of being particularly elementary, a proof which is more concise, and at the same time considerably more general, proceeds effortlessly from Green's theorem. In this and in the remainder of the section we again restrict ourselves to the plane case.

TheOREM 18. Let $\Omega$ be an open set triangulated into a locally finite complex of class $C^{2}$. If $w$ is a function continuous on $\Omega$ and coinciding on each 2-cell $\sigma$ of $\Omega$ with a function of class $C^{2}$ on $\bar{\sigma}$, then $w$ is $\delta$-subharmonic on $\Omega$. Moreover, the mass distribution for $w$ has density $(-1 / 2 \pi) \Delta w$ in the interior of each cell $\sigma$ and density with respect to arc length equal to $1 / 2 \pi$ times the jump of $\partial w / \partial n$ on each boundary curve $\partial \sigma$.

Proof. For any function $f \in C^{2}(\bar{\sigma})$ Green's theorem yields 


$$
\int_{\sigma} w \Delta f d a=\int_{\sigma} f \Delta w d a+\int_{\partial \sigma}[w(\partial f / \partial n)-f(\partial w / \partial n)] d s .
$$

Hence, if $f$ is in $C^{2}(\Omega)$ and vanishes outside of some compact subset, we have

$$
\int_{\Omega} w \Delta f d a=\int_{\Omega} f \Delta w d a-\sum_{\sigma} \int_{\partial \sigma} f(\partial w / \partial n) d s .
$$

It follows by Theorem 11 that $w$ is almost $\delta$-subharmonic and has the asserted mass distribution. That $w$ is actually $\delta$-subharmonic is now evident from the nature of its mass distribution.

REMARK 1. A continuous almost subharmonic function is necessarily subharmonic. Extrapolating from this, one might be tempted to conclude that a continuous almost $\delta$-subharmonic function must be $\delta$-subharmonic. However, as we observe in connection with example 5 , such a conclusion would be false.

A typical method for establishing $\delta$-subharmonicity of a continuous almost $\delta$-subharmonic function is that employed in the above proof: for a continuous almost $\delta$-subharmonic function to be $\delta$-subharmonic it is necessary and sufficient that the potential of its mass distribution on compact subsets be continuous.

To derive analogues of (2) and (3) of Corollary 8.1, we first recall the definition of the upper Blaschke operator in terms of the Blaschke difference operator as

$$
\bar{\Delta}^{B} w=\limsup _{r \rightarrow 0} \Delta_{r}^{B} w .
$$

Replacing lim sup by lim inf gives rise to the lower Blaschke operator. If the upper and lower operators applied to a given function $w$ yield identical functions, then $w$ is said to admit a Blaschke operator, which appears as

$$
\Delta^{B} w=\lim _{r \rightarrow 0} \Delta_{r}^{B} w\left({ }^{11}\right) .
$$

It is well known that whenever $w$ has continuous second partial derivatives at a point, the Blaschke operator exists for $w$ at that point and coincides with the value given by the Laplacian.

Of fundamental importance is the following criterion for subharmonicity (Radó $[1$, p. 14]) : a function $u$ is subharmonic on a region $\Omega$ if and only if $u$ is upper semi-continuous and $\bar{\Delta}^{B} u$ is non-negative on $\Omega$. Whence

THEOREM 19. Let $w$ be a function upper semi-continuous on an open set $\Omega$. If $\bar{\Delta}^{B} w$ is bounded below on compact subsets of $\Omega$, then $w$ is $\delta$-subharmonic on $\Omega$.

(11) Corresponding Privaloff operators are defined similarly in terms of the Privaloff difference operator. Results for the Privaloff operators parallel those for the Blaschke operators. 
Proof. Taking $c$ as a lower bound for $\bar{\Delta}^{B} w$ on a disc $\sigma \subset \Omega$, we define $u$ by $u(x, y)=(1 / 2) c x^{2}$. From the inequality $\bar{\Delta}^{B}(w-u)=\bar{\Delta}^{B} w-\Delta u \geqq 0$ and the upper semi-continuity of $w-u$ it follows that $w-u$ is subharmonic on the interior of $\sigma$. Hence, $w$ is $\delta$-subharmonic.

COROLlaRY 19.1. If w is a continuous function admitting a Blaschke operator bounded on compact subsets of an open set $\Omega$, then $w$ is $\delta$-subharmonic on $\Omega$.

In particular, we recover the classical condition: if $w$ is a function possessing continuous second partial derivatives on an open set $\Omega$, then $w$ is $\delta$-subharmonic on $\Omega$.

This, moreover, admits a further refinement.

COROLlaRY 19.2. Let $w$ be a function admitting partial derivatives of the first order on an open set $\Omega$. A sufficient condition for w to be $\delta$-subharmonic on $\Omega$ is that each point of $\Omega$ lie in a corresponding neighborhood on which $w_{x}$ and $w_{y}$ satisfy a uniform Lipschitz condition of the form

$$
\begin{aligned}
& \left|w_{x}\left(x^{\prime}, y^{\prime}\right)-w_{x}(x, y)\right| \leqq M\left|x^{\prime}-x\right|, \\
& \left|w_{y}\left(x^{\prime}, y^{\prime}\right)-w_{y}(x, y)\right| \leqq M\left|y^{\prime}-y\right| .
\end{aligned}
$$

Proof. Let us employ the notation $z=(x, y)$ and $z^{\prime}=\left(x^{\prime}, y^{\prime}\right)$ for distinct points of a neighborhood on which the above inequalities hold. Defining a remainder function $\eta$ by

$$
w\left(z^{\prime}\right)=w(z)+w_{x}(z)\left(x^{\prime}-x\right)+w_{y}(z)\left(y^{\prime}-y\right)+\eta\left(z^{\prime}, z\right),
$$

we find

$$
\left|\Delta_{r}^{B} w(z)\right| \leqq\left(2 / \pi r^{2}\right) \int_{0}^{2 \pi}\left|\eta\left(z+r e^{i \theta} ; z\right)\right| d \theta .
$$

Since the Lipschitz condition guarantees the continuity of the partial derivatives and thereby of $w$, it suffices to show that the right-hand member is bounded. For this we note that the mean value theorem yields

$$
\eta\left(z^{\prime}, z\right)=\left[w_{x}\left(z^{\prime \prime}\right)-w_{x}(z)\right]\left(x^{\prime}-x\right)+\left[w_{y}\left(z^{\prime \prime}\right)-w_{y}(z)\right]\left(y^{\prime}-y\right)
$$

for some $z^{\prime \prime}$ on the segment joining $z$ and $z^{\prime}$, so that

$$
\left|\eta\left(z^{\prime}, z\right)\right| \leqq M\left|z^{\prime}-z\right|^{2} .
$$

An alternative approach to Corollary 19.2 would be to apply the theory of distributions. In fact, it is readily seen that the Lipschitz condition forces $w_{x x}$ and $w_{y y}$ (taken as distributions) to be locally bounded and hence measures $\left({ }^{12}\right)$. Almost $\delta$-subharmonicity of $w$ then follows from Theorem 10 . However to prove that $w$ is actually $\delta$-subharmonic would require some sort of

(12) Observe that, for $\phi$ an infinitely differentiable function with compact support, $w_{x x}(\phi)$ $=\lim _{h \rightarrow 0} \int \phi(x, y)\left\{\left[w_{x}(x-h, y)-w_{x}(x, y)\right] / h\right\} d a(x, y)$. 
supplementary argument.

In addition to the preceding results attention should be called to the somewhat more delicate sufficient conditions for $\delta$-subharmonicity, in terms of the Blaschke operators, developed by Rudin [1].

While a criterion corresponding to that of Theorem 6 is not available for $\delta$-subharmonicity, a sufficient condition of this sort can be established if we use the Arzelà definition of bounded variation (Adams and Clarkson [1]).

THEOREM 20. Let $w$ be a function on an open set $\Omega$. If each point of $\Omega$ lies in the interior of a corresponding compact cell $K=\left[a, a_{1}\right] \times\left[b, b_{1}\right]$ on which w is given by

$$
w(x, y)=\int_{a}^{x} \int_{b}^{y} f
$$

where $f$ is a function of bounded variation in the sense of Arzelà on $K$, then $w$ is $\delta$-subharmonic on $\Omega$.

Proof. Theorem 7 of Adams and Clarkson [2] allows us to decompose $f$ as $g-h$, where each of the functions $g, h$ is nondecreasing in $y$ for fixed $x$ and nondecreasing in $x$ for fixed $y$. From their convexity properties it follows (Radó $[1$, p. 18]) that the functions $u$ and $v$ defined in the interior of $K$ by

$$
u(x, y)=\int_{a}^{x} \int_{b}^{y} g \text { and } v(x, y)=\int_{a}^{x} \int_{b}^{y} h
$$

are subharmonic. Hence, $w$ is $\delta$-subharmonic.

7. Closure and convergence properties. Many of the basic properties of subharmonic functions remain valid for $\delta$-subharmonic functions. However, the widening of the original class of functions effects two important changes. It extends the closure properties, in fact more than one might at first expect, and it restricts the convergence properties rather severely.

As indicated earlier, a motivation for the study of $\delta$-subharmonic functions is the property of algebraic closure, which we now state explicitly.

THEOREM 21. The family of all functions $\delta$-subharmonic (almost $\delta$-subharmonic, quasi $\delta$-subharmonic) on an open set $\Omega$ constitutes a linear space over the real field with respect to the ordinary operations of addition of functions and of multiplication of functions by reals.

Clearly, the space of all $\delta$-subharmonic functions on $\Omega$ is the space generated by the family of all subharmonic functions on $\Omega$. Note also that we obtain a linear space if we consider the family of all complete $\delta$-subharmonic functions and modify our operations by taking the complete extensions of the resulting $\delta$-subharmonic functions.

Furthermore, under the usual ordering of functions according to functional values, the spaces in Theorem 21 are actually vector lattices. 
THEOREM 22. If $w, w_{1}$, and $w_{2}$ are functions $\delta$-subharmonic (almost $\delta$-subharmonic, quasi $\delta$-subharmonic) on an open set $\Omega$, then $\max \left(w_{1}, w_{2}\right), \min \left(w_{1}, w_{2}\right)$, and $|w|$ are $\delta$-subharmonic (almost $\delta$-subharmonic, quasi $\delta$-subharmonic) on $\Omega$.

Proof. For $w_{1}$ and $w_{2} \delta$-subharmonic the $\delta$-subharmonicity of $\max \left(w_{1}, w_{2}\right)$ hinges on the lattice identity

$$
\max \left(u_{1}-v_{1}, u_{2}-v_{2}\right)=\max \left(u_{1}+v_{2}, u_{2}+v_{1}\right)-\left(v_{1}+v_{2}\right) .
$$

A reflection yields the corresponding property of $\min \left(w_{1}, w_{2}\right)$, while that for $|w|$ results from $|w|=2 w^{+}-w$. In each case the infinities of the various functions are accounted for in obvious fashion.

Thus, the upper and lower envelopes of any finite number of $\delta$-subharmonic functions on $\Omega$ are themselves $\delta$-subharmonic.

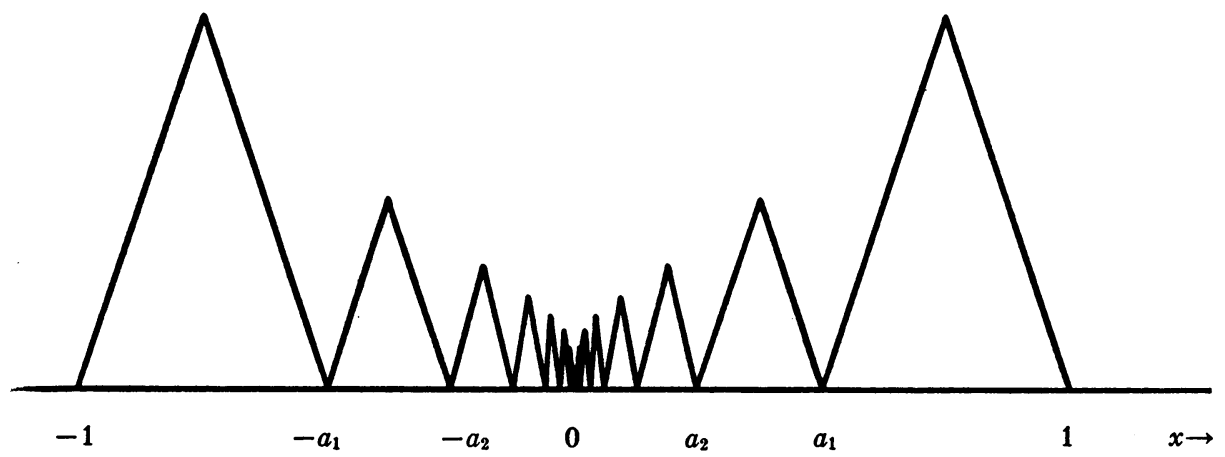

FIG. 1

Passing to the case of the upper envelope of an arbitrary family of such functions, we recall (Brelot $[1$, p. 15]) that in the subharmonic case the upper envelope is subharmonic provided it is upper semi-continuous. Moreover, H. Cartan has shown (Cartan [1, p. 99]) that whenever the family is bounded above, its upper envelope is at least quasi subharmonic.

The situation for $\delta$-subharmonic functions presents a marked contrast.

EXAMPLE 2. There exists an increasing sequence $\left\{w_{k}\right\}$ of continuous $\delta$-subharmonic functions on $\varepsilon^{2}$ converging to a function $w$ which is bounded and continuous but not almost $\delta$-subharmonic. We consider for this a function $f$ continuous on the real axis, vanishing outside of the interval $(-1,1)$, and defining on $(-1,1)$ a continuous polygonal arc of unbounded variation, as indicated in Fig. 1. Then for every positive integer $k$ we define $f_{k}$ as the function which coincides with $f$ outside of $\left(-a_{k}, a_{k}\right)$ and vanishes on $\left(-a_{k}, a_{k}\right)$.

Setting $w_{k}(x, y)=f_{k}(x)$, we find that $\left\{w_{k}\right\}$ converges upward to the function $w$ defined by $w(x, y)=f(x)\left({ }^{13}\right)$. By Theorem 17 each $w_{k}$ is $\delta$-subharmonic.

(13) The sequence can, of course, be made strictly increasing by subtracting $1 / k$ from $w_{k}$ 
However, the theorem of Evans employed in example 1 shows that $w$ is not even almost $\delta$-subharmonic.

A further conclusion to be drawn from this example is that the limit of a uniformly convergent sequence of $\delta$-subharmonic functions need not be almost $\delta$-subharmonic $\left({ }^{14}\right)$. As one might well suspect from the construction employed, the underlying reason for non- $\delta$-subharmonicity of the limit function is to be found in the behavior of the mass distributions of the approximating functions: if $\Omega$ is any open set intersecting the $y$-axis, $\left\{\phi_{\Omega}\left(w_{k}\right)\right\}$ is unbounded.

By stipulating that the mass distributions have uniformly bounded total variations, we arrive at a convergence theorem for almost $\delta$-subharmonic functions. First, however, a few remarks on weak convergence of mass distributions are in order.

Definition 7. Let $\Omega$ be an open set and $\mathcal{C}^{*}$ the set of all functions $f$ continuous on $\Omega$ and tending to 0 on $\partial \Omega$. A sequence $\left\{m_{k}\right\}$ of mass distributions on $\Omega$ will be said to converge weakly on $\Omega$ to a mass distribution $m$ provided

$$
\lim _{k \rightarrow \infty} \int_{\Omega} f d m_{k}=\int_{\mathbf{\Omega}} f d m
$$

for all $f$ in $\mathcal{C}^{\#}$, the indicated integrals being assumed to exist finitely.

Identification of a mass distribution $m$ on $\Omega$ with the linear functional

$$
\Lambda(f)=\int_{\Omega} f d m
$$

reveals that weak convergence of $\left\{m_{k}\right\}$ to $m$ is simply weak convergence of linear functionals on the Banach space $\mathcal{C}^{*}$ (under the usual norm, $\|f\|$ $=\max |f|$ ). In dealing with weak convergence unstinting use is made of the theorems of $\$ 4$, pp. 122-123, of Banach [1] and of the fact (Hewitt [1, p. 459]) that

$$
\|\Lambda\|=|m|(\Omega) .
$$

The separability of $\mathcal{C}^{\#}$ and the density in $\mathcal{C}^{*}$ of the spaces $\mathcal{C}^{2}$ and $\mathcal{C}^{*}$ (appearing in the proof of Theorem 11) also play important roles. Replacing $\mathcal{C}^{*}$ by $\mathcal{C}$ in Definition 7 yields the notion of vague convergence according to $\mathrm{H}$. Car$\tan [1, \mathrm{p} .77]$. Clearly, weak convergence is equivalent to the combination of vague convergence and boundedness of the total variations of the mass distributions.

The above remarks find application in the proof of our convergence theorem.

THEOREM 23. Let $\Omega$ be an open set and $\left\{w_{k}\right\}$ a sequence of functions almost

(14) The fact that $\left\{w_{k}\right\}$ happens to converge uniformly to $w$ is not really essential here. Any continuous function can be uniformly approximated on compact sets by $\delta$-subharmonic functions (in particular by polynomials). 
$\delta$-subharmonic on $\Omega$. If the sequence $\left\{\phi_{\Omega}\left(w_{k}\right)\right\}$ of total variations of the mass distributions is bounded and $\left\{w_{k}\right\}$ converges in the mean to a function $w$ on $\Omega$, then

(1) $w$ is almost $\delta$-subharmonic on $\Omega$, and

(2) the sequence $\left\{m_{k}\right\}$ of mass distributions for the functions $w_{k}$ converges weakly to the mass distribution $m$ for $w$.

Proof. The boundedness of $\left\{\phi_{\Omega}\left(w_{k}\right)\right\}$ implies the existence of a subsequence of $\left\{m_{k}\right\}$ converging weakly on $\Omega$ to a mass distribution $m$, and for the sake of notational simplicity we take this subsequence as $\left\{m_{k}\right\}$ itself. Furthermore, there is no loss of generality in supposing $\Omega$ to be a bounded Dirichlet region, thus admitting a Green's function $G$. We then introduce the $\delta$-subharmonic functions

$$
W_{k}(z)=\int_{\Omega} G(z, \zeta) d m_{k}(\zeta) \text { and } W(z)=\int_{\Omega} G(z, \zeta) d m(\zeta)
$$

and note that $w_{k}=W_{k}+H_{k}$, where $H_{k}$ is harmonic on $\Omega$.

The function

$$
G_{r}(z, \zeta)=\left(1 / \pi r^{2}\right) \int_{0}^{r} \int_{0}^{2 \pi} G\left(z+\rho e^{i \theta}, \zeta\right) \rho d \theta d \rho,
$$

appearing as the integrand in the expressions

$$
\alpha_{r} W_{k}(z)=\int_{\Omega} G_{r}(z, \zeta) d m_{k}(\zeta) \quad \text { and } \quad \alpha_{r} W(z)=\int_{\Omega} G_{r}(z, \zeta) d m(\zeta),
$$

is continuous on $\zeta$ for fixed $r$ and $z$, and coincides with $G(z, \zeta)$ for $\zeta$ outside of $N_{r}(z)$. Thus, the weak convergence of $\left\{m_{k}\right\}$ to $m$, together with the continuous vanishing in $\zeta$ of $G(z, \zeta)$ on the boundary of $\Omega$, yields

$$
\lim _{\boldsymbol{k} \rightarrow \infty} \alpha_{r} W_{k}=\alpha_{r} W \text {. }
$$

Also, by the mean convergence of $\left\{w_{k}\right\}$ to $w$ we have

$$
\lim _{k \rightarrow \infty} \alpha_{r} w_{k}=\alpha_{r} w,
$$

from which it follows that $\left\{H_{k}\right\}$ converges to a function $H$ continuous on $\Omega$ and that $\alpha_{r} w=\alpha_{r} W+H$. A duplication of the preceding argument results in $\alpha_{r} H=H$, proving that $H$ is harmonic. In the limit as $r \rightarrow 0$ we obtain $w$ $=W+H$ almost everywhere on $\Omega$, so that $w$ is almost $\delta$-subharmonic.

Having ascertained the almost $\delta$-subharmonicity of $w$, we infer without difficulty from (5.2) of Theorem 11 that the original sequence of mass distributions converges weakly on $\Omega$ to $m$.

The following strengthening of conclusion (2) seems noteworthy.

THEOREM 24. Let $\mathcal{F}$ be a bounded equicontinuous family of functions on the 
open set $\Omega$, such that all functions in $\mathcal{F}$ vanish outside a fixed compact subset of $\Omega$. Then, under the hypotheses of Theorem 23

$$
\lim _{k \rightarrow \infty} \int_{\Omega} f d m_{k}=\int_{\Omega} f d m \quad \text { uniformly for } f \text { in } \mathcal{Z} \text {. }
$$

Proof. Let us take $f$ as any function in $\mathcal{F}$, and $F$ as the double areal mean $F=\alpha_{r} \alpha_{r} f$. Since $F$ is in $C^{2}(\Omega)$ and (for $r$ sufficiently small) vanishes outside of a compact subset of $\Omega$, we see from (5.2) that

$$
\left|\int_{\Omega} F d m_{k}-\int_{\Omega} F d m\right| \leqq(1 / 2 \pi) \max |\Delta F| \int_{\Omega}\left|w_{k}-w\right| d a .
$$

Moreover, the evident inequalities

$$
\left|\Delta\left(\alpha_{r} \alpha_{r} f\right)\right| \leqq\left(4 / r^{2}\right) \max |f|
$$

and

$$
\left|\alpha_{r} \alpha_{r} f-f\right| \leqq \omega_{f}(2 r),
$$

where $\omega_{f}$ denotes the modulus of continuity of $f$, lead to

$$
\left|\int_{\Omega} f d m_{k}-\int_{\Omega} f d m\right| \leqq 2 \omega_{f}(2 r) M+\left(2 / \pi r^{2}\right) \max |f| \int_{\Omega}\left|w_{k}-w\right| d a
$$

with $M$ taken as any upper bound for the total variations of the mass distributions. Uniformity of the convergence is now obvious.

We examine next the extent to which the multiplicative closure property of Corollary 8.2 carries over to higher dimensions. A direct analogue of this corollary is readily obtained by algebraic methods, provided we impose on the functions involved certain boundedness restrictions automatically fulfilled in the 1-dimensional case.

THEOREM 25. The space of $\delta$-subharmonic functions on an open set $\Omega$ generated by the family of all locally bounded subharmonic functions on $\Omega$ is an algebra (that is, it is closed under the operation of multiplication of functions).

Proof. We wish to show that the product of any two functions $w_{1}$ and $w_{2}$ of the space can be represented as the difference of two locally bounded subharmonic functions, and the identity $w_{1} w_{2}=(1 / 4)\left[\left(w_{1}+w_{2}\right)^{2}-\left(w_{1}-w_{2}\right)^{2}\right]$ reduces this to the case of $w_{1}=w_{2}$. Then, starting with $w=u-v$, where $u$ and $v$ are locally bounded subharmonic functions, we have $w^{2}=2\left(u^{2}+v^{2}\right)-(u+v)^{2}$. From this and the fact that the square of a positive subharmonic function is subharmonic (Radó [1, p. 19]) it follows that $w^{2}$ is $\delta$-subharmonic and that the subharmonic functions appearing in the canonical representation for $w$ are bounded.

REMARK 2. In connection with the statement of Theorem 25 it should be 
mentioned that a bounded $\delta$-subharmonic function may fail to admit a representation as the difference of two locally bounded subharmonic functions. (See example 3.)

However, in contrast with the $\delta$-convex situation it is not true that the product of two arbitrary $\delta$-subharmonic functions is $\delta$-subharmonic, or even almost $\delta$-subharmonic. For example, $u^{2}$, where $u(z)=\log |z|$, is not almost $\delta$-subharmonic on any neighborhood of the origin, since the concentrated mass at $z$ for any $\delta$-subharmonic function $w$ is given by $\lim _{r \rightarrow 0}\left[\mu_{r} w(z) /(-\log r)\right]$.

Turning our attention to the space of almost $\delta$-subharmonic functions, we show that the local boundedness requirement in Theorem 25 can be removed for one of the factors whenever the other factor is sufficiently well behaved.

THEOREM 26. Let $\Omega$ be an open set and $S$ the space of $\delta$-subharmonic functions generated by the family of all subharmonic functions on $\Omega$ which are locally bounded and have locally essentially bounded first partial derivatives. If $w_{1}$ and $w_{2}$ are almost $\delta$-subharmonic functions on $\Omega$ one of which coincides almost everywhere with a function in $S$, then $w_{1} w_{2}$ is almost $\delta$-subharmonic on $\Omega$.

Proof. There is obviously no loss of generality in assuming $w_{1}$ and $w_{2}$ to be subharmonic. By Theorem 25 it then suffices to prove that $u v$ is almost $\delta$-subharmonic, where $u$ is a bounded subharmonic function having essentially bounded first partial derivatives and $v$ is the potential of a negative mass distribution $m$.

For convenience we suppose that $S_{1 / 2}(0) \subset \Omega$ and that the mass of $v$ lies entirely on $\omega=N_{1 / 2}(0)$. Let us denote by $U_{\rho}$ the triple areal mean of $u$ over circles of radius $\rho$ and for $z[=(x, y)]$ and $\zeta[=(\xi, \eta)]$ on $\omega$ define

$$
W_{\rho, r, \zeta}(z)=U_{\rho}(z) L_{r}(z, \zeta),
$$

where

$$
L_{r}(z, \zeta)=\max (\log |z-\zeta|, \log r) .
$$

By Theorem 25, $W_{\rho, r, \xi}$ is $\delta$-subharmonic on $\omega$. This is, however, also a consequence of Theorem 18, which asserts further that the mass of $W_{\rho, r, \zeta}$ is given by a distribution of density $(-1 / 2 \pi) \Delta W_{\rho, r, \zeta}$ on $\omega-C_{r}(\zeta)$ and of linear density $1 / 2 \pi$ times the jump of $\partial W_{\rho, r, \zeta} / \partial n$ on $\omega \cap C_{r}(\zeta)$.

Let us determine estimates for the total variation of this mass distribution. Direct computation yields

$$
\begin{array}{rlr}
\Delta W_{\rho, r, \zeta}(z)= & 2\left[(x-\xi) \frac{\partial U_{\rho}(z)}{\partial x}+(y-\eta) \frac{\partial U_{\rho}(z)}{\partial y}\right] /|z-\zeta|^{2} \\
& +\left[\Delta U_{\rho}(z)\right] \log |z-\zeta| & \text { on } \omega-S_{r}(\zeta) \\
= & {\left[\Delta U_{\rho}(z)\right] \log r} & \text { on } \omega \cap N_{r}(\zeta)
\end{array}
$$


and shows the jump of $\partial W_{\rho, r, \zeta} / \partial n$ to be $U_{\rho}(z) / r$ on $\omega \cap C_{r}(\zeta)$. The total variation is then given by

$$
\phi_{\omega}\left(W_{\rho, r, \zeta}\right)=(1 / 2 \pi) \int_{\omega-C_{r}(\zeta)}\left|\Delta W_{\rho, r, \zeta}\right| d a+(1 / 2 \pi r) \int_{\omega \cap C_{r}(\zeta)}\left|U_{\rho}\right| d s .
$$

Taking $M$ as a bound for $|u|$ and (except for a set of measure zero) for $\left|u_{x}\right|$ and $\left|u_{y}\right|$, we see that $M$ also bounds $\left|U_{\rho}\right|,\left|\partial U_{\rho} / \partial x\right|$, and $\left|\partial U_{\rho} / \partial y\right|$. Now, the choice of $1 / 2$ as radius of $\omega$ ensures $\log r<0$ and $\log |z-\zeta|<0$, so that

$$
\begin{aligned}
(1 / 2 \pi) \int_{\omega-C_{r}(\zeta)}\left|\Delta W_{\rho, r, \zeta}\right| d a \leqq & (M / \pi) \int_{\omega} \frac{|x-\xi|+|y-\eta|}{|z-\zeta|^{2}} d a(z) \\
& -(1 / 2 \pi) \int_{\omega}\left[\Delta U_{\rho}(z)\right] \log |z-\zeta| d a(z) \\
& -(1 / 2 \pi)(\log r) \int_{\omega \cap N_{r}(\zeta)} \Delta U_{\rho} d a .
\end{aligned}
$$

By Green's theorem the final term in the right-hand member is seen to be dominated by $-2^{1 / 2} \mathrm{Mr} \log r$. Also, since the second term in the right-hand member is the negative of the potential at $\zeta$ of the mass distribution for $U_{\rho}$, it is apparent that the left-hand member is bounded for all $\zeta \in \omega$ and all sufficiently small $\rho$ and $r$. From the obvious boundedness of the remaining integral in $\left(^{*}\right)$ we infer the existence of a constant $K$ such that $\phi_{\omega}\left(W_{\rho, r, \zeta}\right) \leqq K$.

Letting $\rho \rightarrow 0$ results in convergence in the mean of $U_{\rho}$ to $u$. It follows by Theorem 25 that the function $w_{r, 5}$ defined on $\omega$ by

$$
w_{r, \zeta}(z)=u(z) L_{r}(z, \zeta)
$$

is almost $\delta$-subharmonic and that $\phi_{\omega}\left(w_{r, \xi}\right) \leqq K$. We use next the fact that $u v$ is the limit in the mean of a sequence $\left\{s_{k}\right\}$ of finite sums of the form

$$
s_{k}(z)=-u(z) \sum_{j} L_{1 / k}\left(z, \zeta_{j}\right) m\left(e_{j}\right)
$$

where $\left\{e_{j}\right\}$ is a partition of $\omega$. Almost $\delta$-subharmonicity of $u v$ now follows from the evident inequality $\phi_{\omega}\left(s_{k}\right) \leqq K \phi_{\omega}(v)$.

A somewhat different proof of this theorem can be given by approximating $u$ and $v$ by functions having continuous second partial derivatives, investigating convergence for the various terms obtained by taking the Laplacian of the product of the approximants, and concluding that the $\Delta$ of Schwartz for $u v$ is a measure.

In particular, the product of a harmonic function and an almost $\delta$-subharmonic function is almost $\delta$-subharmonic. This fact can be utilized to advantage to obtain a concise proof for the $\delta$-subharmonic analogue of the 
absolute continuity of $\delta$-convex functions, a result due to Evans [1, p. 45] who applied the theory of potential functions of generalized derivatives.

THEOREM 27. Every continuous almost $\delta$-subharmonic function is absolutely continuous in the sense of Tonelli( $\left.{ }^{15}\right)$.

Proof. Let $w$ be a continuous almost $\delta$-subharmonic function on an open set $\Omega$, and let $\sigma$ be a disc lying in $\Omega$. Defining $W$ by $W(x, y)=x w(x, y)$, we find by a simple calculation

$$
\Delta_{r}^{B} W(z)=x \Delta_{r}^{B} w(z)+(2 / \pi r) \int_{0}^{2 \pi} w\left(z+r e^{i \theta}\right) \cos \theta d \theta .
$$

The final term here can be expressed in terms of the areal mean $\alpha_{r} w$ as $2\left[\partial \alpha_{r} w(z) / \partial x\right]$, and we have

$$
2\left|\partial \alpha_{r} w(z) / \partial x\right| \leqq\left|\Delta_{r}^{B} W(z)\right|+|x| \cdot\left|\Delta_{r}^{B} w(z)\right| .
$$

Since $W$ and $w$ are both almost $\delta$-subharmonic, it follows from Theorem 14 that

$$
\limsup _{r \rightarrow 0} \int_{\sigma}\left|\partial \alpha_{r} w / \partial x\right| d a<+\infty .
$$

The same being true for the derivative with respect to $y$, we conclude (Saks $[1$, p. 179]) that $w$ has finite area over $\sigma$. Evans has shown (Radó [1, p. 45]) that $w$ is absolutely continuous in $x$ for almost all $y$ and absolutely continuous in $y$ for almost all $x$. Hence, $w$ is absolutely continuous in the sense of Tonelli.

As a consequence, the surface defined by a continuous almost $\delta$-subharmonic function $w$ has finite area over any closed disc $\sigma$ lying in the domain of $w$, and the area $S$ is given by the classical formula

$$
S=\int_{\sigma}\left[\left(w_{x}\right)^{2}+\left(w_{y}\right)^{2}+1\right]^{1 / 2} d a .
$$

The finiteness of the area, which is of course the key to the above proof, stems from the finiteness of the area of $\log |z|$ over any disc. On this basis one can establish (7.1) by a direct calculation for $w$ a potential and thereby obtain a more elementary, but somewhat less concise, proof of the theorem.

A further closure property which we consider involves the operation of composition of functions. Given a $\delta$-subharmonic function $w$ and a real function $f$ whose domain contains the range of $w$, we can form the composite function $W=f \circ w$ (that is, the function defined on the domain of $w$ by $W(z)$

(15) See Saks [1, p. 169ff.] for the definition of, and properties related to, this type of absolute continuity. 
$=f[w(z)])$. Under certain conditions on $f$ and $w$ the resulting composite function will be $\delta$-subharmonic, or at least almost $\delta$-subharmonic.

For example, Theorem 25 assures us that $W$ is $\delta$-subharmonic whenever $w$ is the difference of two locally bounded subharmonic functions and $f$ is a polynomial. However, as is to be expected, almost $\delta$-subharmonicity of $W$ can be established under considerably weaker hypotheses on $f$.

TheOREM 28. Let $w$ be a function almost $\delta$-subharmonic on an open set $\Omega$ and admitting a representation $(u, v)$, where $u$ and $v$ are bounded subharmonic functions. Further, let $f$ be a real function having a Lipschitzian derivative on an interval $I$ containing the range of $w$. Then the composite function $W=f \circ w$ is almost $\delta$-subharmonic on $\Omega$.

Proof. For some $M>0$ the function $f$ satisfies

$$
\left|f^{\prime}(t)-f^{\prime}(s)\right| \leqq M|t-s|
$$

for all $s, t$ on $I$. Then, defining a remainder function $\eta$ by

$$
f(t)=f(s)+f^{\prime}(s)(t-s)+\eta(s, t),
$$

we see from the mean-value theorem that

$$
|\eta(s, t)| \leqq M|t-s|^{2} .
$$

A simple calculation yields

$$
\begin{aligned}
\left|\Delta_{r}^{B} W(z)\right| \leqq & \left|f^{\prime}[w(z)]\right| \cdot\left|\Delta_{r}^{B} w(z)\right| \\
& +\left(2 M / \pi r^{2}\right) \int_{0}^{2 \pi}\left[w\left(z+r e^{i \theta}\right)-w(z)\right]^{2} d \theta,
\end{aligned}
$$

and it is convenient here to transform the final term by means of the identity

$$
\begin{aligned}
{\left[w\left(z+r e^{i \theta}\right)-w(z)\right]^{2}=} & w^{2}\left(z+r e^{i \theta}\right)-w^{2}(z) \\
& -2 w(z)\left[w\left(z+r e^{i \theta}\right)-w(z)\right] .
\end{aligned}
$$

There results

$$
\left|\Delta_{r}^{B} W\right| \leqq\left(\left|f^{\prime} \circ w\right|+2 M|w|\right) \cdot\left|\Delta_{r}^{B} w\right|+M \cdot\left|\Delta_{r}^{B} w^{2}\right| .
$$

Since $f^{\prime}$ and $w$ are bounded and $w$ and $w^{2}$ are almost $\delta$-subharmonic, Theorem 14 shows that $W$ is almost $\delta$-subharmonic.

Having observed that the Lipschitz condition on $f^{\prime}$ forces $f$ to be $\delta$-convex, one may well ask whether anything more than $\delta$-convexity is really needed. We leave this question open in the general case, but answer it in the negative for $w$ restricted in a manner reminiscent of Theorem 26 .

THEOREM 29. Let $w$ be an almost $\delta$-subharmonic function which is bounded and has essentially bounded first partial derivatives on an open set $\Omega$. If $[a, b]$ 
is an interval containing the range of $w$ and $f$ is a function $\delta$-convex on $[a-\epsilon$, $b+\epsilon]$ for some $\epsilon>0$, then $W=f \circ w$ is almost $\delta$-subharmonic on $\Omega$.

Proof. Without loss of generality we can suppose $f$ to be an increasing convex function, linear on $[a-\epsilon, a]$ and $[b, b+\epsilon]$. We then fix $\sigma$ as any closed disc contained in $\Omega$ and define $w_{\rho}$ on $\sigma$ as the triple areal mean of $w$ over circles of radius $\rho: w_{\rho}=\alpha_{\rho} \alpha_{\rho} \alpha_{\rho} w$. Similarly, $f_{\rho}$ will be defined as a double mean for $f$ :

$$
f_{\rho}(t)=\left(1 / 4 \rho^{2}\right) \int_{t-\rho}^{t+\rho}\left(\int_{s-\rho}^{s+\rho} f\right) d s .
$$

Setting $W_{\rho}=f_{\rho} \circ w_{\rho}$, we find

$$
\left|\Delta W_{\rho}\right| \leqq\left(f_{\rho}^{\prime} \circ w_{\rho}\right)\left|\Delta w_{\rho}\right|+\left(f_{\rho}^{\prime \prime} \circ w_{\rho}\right)\left[\left(\partial w_{\rho} / \partial x\right)^{2}+\left(\partial w_{\rho} / \partial y\right)^{2}\right] .
$$

It is clear that for small $\rho$ the functions $f_{\rho}^{\prime}$ are uniformly bounded above by some number $M$, and there follows

$$
\begin{aligned}
\int_{\sigma}\left|\Delta W_{\rho}\right| d a \leqq & M \int_{\sigma}\left|\Delta w_{\rho}\right| d a \\
& +\int_{\sigma}\left(f_{\rho}^{\prime \prime} \circ w_{\rho}\right)\left[\left(\partial w_{\rho} / \partial x\right)^{2}+\left(\partial w_{\rho} / \partial y\right)^{2}\right] d a .
\end{aligned}
$$

Now, an application of Lebesgue's bounded convergence theorem shows that $W_{\rho} \rightarrow W$ in the mean as $\rho \rightarrow 0$. Moreover, $\int_{\sigma}\left|\Delta w_{\rho}\right| d a$ remains bounded. Our theorem will therefore be proved if we can establish boundedness of the final integral. To this end we set $F_{\rho}=f_{\rho}^{\prime} \circ w_{\rho}$ and observe that the term in question becomes

$$
\int_{\sigma}\left[\left(\partial F_{\rho} / \partial x\right)\left(\partial w_{\rho} / \partial x\right)+\left(\partial F_{\rho} / \partial y\right)\left(\partial w_{\rho} / \partial y\right)\right] d a,
$$

which Green's theorem permits us to express as

$$
-\int_{\sigma} F_{\rho} \Delta w_{\rho} d a+\int_{\partial_{\sigma}} F_{\rho}\left(\partial w_{\rho} / \partial n\right) d s \leqq M \int_{\sigma}\left|\Delta w_{\rho}\right| d a+M \int_{\partial_{\sigma}}\left|\partial w_{\rho} / \partial n\right| d s .
$$

By virtue of the essential boundedness of $w_{x}$ and $w_{y}, \partial w_{\rho} / \partial n$ is uniformly bounded, and the theorem follows.

Spaces of $\delta$-subharmonic and almost $\delta$-subharmonic functions can in general be normed in a wide variety of ways. We indicate here a norm which is closely related to convergence in the mean and gives rise to a Banach space.

THEOREM 30. Let $\Omega$ be an open set and $\mathbb{B}$ the space of all summable almost $\delta$-subharmonic functions $w$ on $\Omega$ such that $\phi_{\Omega}(w)<+\infty$. Then 


$$
\|w\|=\phi_{\Omega}(w)+\int_{\Omega}|w| d a
$$

defines a norm under which $B$ is a Banach space.

Proof. The norm properties are readily verified, and completeness is an easy consequence of Theorem 23.

Banach spaces of $\delta$-subharmonic functions under a slightly different norm will be discussed in a later paper dealing with functions of potential type.

8. Some specific decompositions. A problem of interest, but one which is on the whole rather difficult, is that of determining when a given $\delta$-subharmonic function possessing a property $P$ can be decomposed as the difference of two subharmonic functions both possessing the property $P$.

For example, it is well known that a polynomial of degree $n$ on a bounded region can be represented as the difference of two subharmonic polynomials of degree at most $n{ }^{(16)}$. We show now that if the restriction on the degrees of the representing polynomials is relaxed, the result remains valid for unbounded regions.

THEOREM 31. Every polynomial on $\mathcal{E}^{2}$ can be represented as the difference of two polynomials subharmonic throughout $\varepsilon^{2}$.

Proof. It clearly suffices to show that each term of the given polynomial admits such a representation, and there is no loss of generality in supposing that the generic term under consideration has coefficient one.

Taking first the terms which are of degree at least two in each variable, we have a polynomial of the type $w(x, y)=x^{p+2} y^{q+2}$, where $p$ and $q$ are nonnegative integers. The polynomials

$$
u(x, y)=k x^{2 p+2} y^{2}+x^{p+2} y^{q+2}+x^{2} y^{2 q+2}
$$

and

$$
v(x, y)=k x^{2 p+2} y^{2}+x^{2} y^{2 q+2}
$$

have the evident property that $w=u-v$, and we show that for $k$ sufficiently large both $u$ and $v$ are subharmonic on $\varepsilon^{2}$. This is immediate for $v$, whose Laplacian contains only even powers of $x$ and $y$. For $u$ we compute the Laplacian as

$$
\begin{aligned}
\Delta u(x, y)= & y^{2}\left[k(2 p+2)(2 p+1) x^{2 p}+(p+2)(p+1) x^{p} y^{q}+2 y^{2 q}\right] \\
& +x^{2}\left[2 k x^{2 p}+(q+2)(q+1) x^{p} y^{q}+(2 q+2)(2 q+1) y^{2 q}\right]
\end{aligned}
$$

and observe that both of the bracketed quadratic forms in $x^{p}, y^{q}$ are positive definite provided $k$ is chosen sufficiently large.

$\left.{ }^{16}\right)$ The proof consists simply of translating the given region into the first quadrant and noting that those terms with positive coefficients are subharmonic, while those with negative coefficients are superharmonic. 
Terms of degree one in $x$ and of degree one or less in $y$ are obviously harmonic, so we need only consider terms of degree one in $x$ which are of the form $w(x, y)=x y^{q+2}$, where $q$ is a non-negative integer. The desired decomposition here is obtained by setting $u(x, y)=k x^{2} y^{2}+x y^{q+2}+y^{2 q+2}$ and $v(x, y)$ $=k x^{2} y^{2}+y^{2 q+2}$ and applying the previous argument. Similarly, the term $w(x, y)=y^{q+2}$ of degree zero in $x$ can be expressed as the difference of

$$
u(x, y)=k y^{2}+y^{q+2}+y^{2 q+2} \text { and } v(x, y)=k y^{2}+y^{2 q+2} .
$$

The next property which we consider is based on the following generalization of Poisson's equation: a necessary and sufficient condition for a function $w \delta$-subharmonic on an open set $\Omega$ to have its mass distribution given by a continuous density function $\rho$ is that $w$ be continuous and admit a continuous Blaschke operator $\left({ }^{17}\right)$ on $\Omega$; when this condition is satisfied, $\Delta^{B} w=-2 \pi \rho$. The necessity has been demonstrated by Privaloff [2], and the sufficiency is readily established.

THEOREM 32. If $w$ is a continuous function admitting a continuous Blaschke operator on an open set $\Omega$, then the canonical representation for $w$ consists of two continuous subharmonic functions admitting continuous Blaschke operators on $\Omega\left({ }^{17}\right)$.

Proof. We observe simply that the mass distributions for these subharmonic functions are given by the continuous density functions $(1 / 2 \pi)\left[\Delta^{B} w\right]^{-}$ and $(-1 / 2 \pi)\left[\Delta^{B} w\right]^{+}$.

If the property under consideration is weakened to be merely that of continuity, then, as the following counterexample shows, it is no longer generally preserved under decomposition.

EXAMPLE 3. We construct a bounded continuous potential $w$ on $\varepsilon^{2}$ for which every representation $(u, v)$ has the property that both $u$ and $v$ are unbounded at the origin. To accomplish this, we consider the circles $C_{r_{k}}(0)$ with radii $r_{k}=e^{-k}$ for all positive integers $k$. If the mass $-1 / k^{3}$ is distributed on $C_{r_{k}}(0)$ in any manner whatsoever, then the total mass taken over all the circles is finite and the potential of the resulting mass distribution is finite at the origin. The actual distribution of mass on $C_{r_{k}}(0)$ which we employ is a uniform distribution on a subarc $\alpha_{k}$ with midpoint at $\left(r_{k}, 0\right)$ and with length so small that the potential $u_{k}$ due to this distribution has a value $\leqq-k$ at $\left(r_{k}, 0\right)$.

It is well known from classical potential theory that the potential of a continuous line distribution is continuous. Hence, $u_{k}$ is uniformly continuous on $S_{1}(0)$, and there exists a positive number $t_{k}$ less than $r_{k}-r_{k-1}$ such that $\left|u_{k}(z)-u_{k}\left(z+t_{k}\right)\right| \leqq 1 / k^{2}$ for $z$ on $S_{1}(0)$. Defining $v_{k}$ as the potential of the mass $-1 / k^{3}$ distributed uniformly on the arc $\beta_{k}$ obtained by translating $\alpha_{k}$ a distance $t_{k}$ to the left, we see that $v_{k}(z)=u_{k}\left(z+t_{k}\right)$.

(17) A corresponding theorem holds for the Privaloff operator. 
If $w$ is the potential due to the given distributions on all the arcs $\alpha_{k}$ and the negative of the given distributions on all the $\operatorname{arcs} \beta_{k}$, then it is apparent that the series $\sum_{k=1}^{\infty}\left(u_{k}-v_{k}\right)$ converges uniformly on $S_{1}(0)$ to $w$. Hence, $w$ is continuous on $\mathcal{E}^{2}$, and since it vanishes at infinity, $w$ is also bounded. We see, however, from the definition of the mass distribution for $w$ that all representations of $w$ consist of subharmonic functions unbounded at the origin.

9. Local behavior of $\delta$-subharmonic functions. Possibilities for the local behavior of $\delta$-subharmonic functions are considerably more complicated than for subharmonic functions and give rise to marked distinctions between the two theories. We proceed to examine a few of these possibilities.

The following lemma is fundamental in the construction of some important examples.

Lemma 2. Let $\left\{a_{k}\right\}$ be an alternating sequence of real numbers for which $\sum_{k=1}^{\infty}\left|a_{k}\right|=\infty$. Then there exist concave strictly increasing functions $f_{1}$ and $f_{2}$ on $[0, \infty)$ with the following properties:

(1) $f_{1}(0)=f_{2}(0)=0$,

(2) $\lim _{\infty} f_{1}=\lim _{\infty} f_{2}=\infty$,

(3) $\lim _{x \rightarrow \infty}\left[f_{1}(x) / x\right]=\lim _{x \rightarrow \infty}\left[f_{2}(x) / x\right]=0$,

(4) $f_{1}$ and $f_{2}$ are piecewise linear, and

(5) the piecewise linear function $f=f_{1}-f_{2}$ has alternate maximum and minimum values given by the sequence $\left\{a_{k}\right\}$.

Proof. We indicate graphically the construction of $f_{1}$ and $f_{2}$ as polygonal arcs issuing from the origin and formed by successive segments $p_{1}, p_{2}, p_{3}, \cdots$ and $q_{1}, q_{2}, q_{3}, \cdots$, respectively. Taking the slope of $p_{1}$ as 2 and that of $q_{1}$ as 1 , we proceed on these segments until $f_{1}-f_{2}$ attains the value $a_{1}(>0)$. Then the slope of $f_{1}$ is reduced to $1 / 2$, and we proceed on the segments $p_{2}$ and $q_{1}$ until $f_{1}-f_{2}$ attains the value $a_{2}(<0)$. At this point the slope of $f_{2}$ is reduced to $1 / 4$ and the process repeated. Carrying this on ad infinitum, we arrive at the definition of $f_{1}$ and $f_{2}$.

Properties (1), (4), and (5) are immediate, and for (2) we make use of the divergence of $\sum_{k=1}^{\infty}\left|a_{k}\right|$. Property (3) follows from the fact that the slopes of the segments forming the graphs of $f_{1}$ and $f_{2}$ tend downward to zero, so that $f_{1}$ and $f_{2}$ are ultimately dominated by linear functions of arbitrarily small slope.

Since a convex function of a harmonic function is subharmonic (Radó [1, p. 16]), the functions defined by $-f_{1}(-\log |z|)$ and $-f_{2}(-\log |z|)$ are subharmonic on $N_{1}(0)-(0)$. Furthermore, both of these functions have limit $-\infty$ at 0 , so that we can extend them to be subharmonic throughout $N_{1}(0)$. It follows that the function $w$ defined on $N_{1}(0)$ by $w(z)=f(-\log |z|)$ is $\delta$-subharmonic. Also, since $\lim _{r \rightarrow 0}\left[\mu_{r} w(0) / \log r\right]=0, w$ has no concentrated mass at the origin. By an appropriate choice of the successive peaks, we obtain 
EXAmple 4. There exists a $\delta$-subharmonic function on $N_{1}(0)$ whose circumferential mean on $C_{r}(0)$ does not tend to a limit as $r \rightarrow 0$.

The following example is noteworthy because it shows (as pointed out already in Remark 2) that a continuous almost $\delta$-subharmonic function may fail to be $\delta$-subharmonic.

EXAmple 5. There exists a bounded complete $\delta$-subharmonic function $w$ on $N_{1}(0)$ such that $w(z)$ tends to a finite limit as $z \rightarrow 0$ but $w$ itself is undefined at 0 . Here we set $a_{k}=(-1)^{k-1} / k$, which ensures the existence of the limit. However, since $\mu_{r} w(0)$ is not of bounded variation in $r$ on any interval $(0, R)$, we see that no definition of $w$ at 0 can yield a $\delta$-subharmonic function.

It is a trivial matter to obtain from $w$ a bounded $\delta$-subharmonic function $w^{*}$ on $N_{1}(0)$ such that $w^{*}(z)$ does not tend to a limit as $z \rightarrow 0$, but $\lim _{r \rightarrow 0} \mu_{r} w^{*}(0)$ $=0$ and $w^{*}$ is complete but undefined at 0 . We need only add to $w$ a bounded subharmonic function assuming the value 0 at the origin but discontinuous there.

In view of the existence of a pseudo limit $\left({ }^{18}\right)$ for any subharmonic function at each point of its domain, it is natural to consider questions involving the pseudo limit in the $\delta$-subharmonic case. From the subharmonic result it is obvious that any $\delta$-subharmonic function has a pseudo limit at every point at which it is defined, finitely or infinitely. However, the function appearing in Example 4 shows that a $\delta$-subharmonic function on an open set $\Omega$ need not have a pseudo limit at all points of $\Omega$.

On the other hand, assuming the existence of a pseudo limit, we can under certain conditions infer properties of the corresponding means.

THEOREM 33. If $w$ is a bounded $\delta$-subharmonic function on an open set $\Omega$, admitting a pseudo limit $A$ at $z \in \Omega$, then

$$
\lim _{r \rightarrow 0} \mu_{r} w(z)=A \text {. }
$$

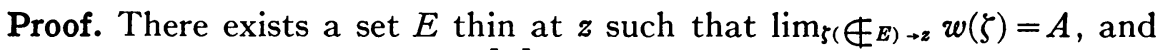
by the theory of thin sets (Brelot [5]) there exists a finite subharmonic function $v$ such that $v(z)=0$ and $\lim _{\zeta(\in E) \rightarrow z} v(\zeta)=-\infty$. Hence $\lim \sup _{z}(w+v)=A$, so that $\lim \sup _{r \rightarrow 0} \mu_{r} w(z) \leqq A$. A similar argument yields the reversed inequality for the limit inferior, and the theorem follows.

Of course, whenever the circumferential mean has limit $A$, the areal mean likewise has a limit $A$.

In dropping the boundedness condition, we consider the areal mean rather than the circumferential mean and note a fundamental distinction between the 2-dimensional and higher dimensional cases.

Lemma 3. Let $E$ be a measurable subset of $\mathcal{E}^{2}$ thin at 0 , and denote by $E_{r}$ the intersection of $E$ with $N_{r}(0)$. If $w$ is any function almost $\delta$-subharmonic in

(18) For a discussion of pseudo limits and thin sets see Brelot [5]. 
the neighborhood of 0 , then

$$
\lim _{r \rightarrow 0}\left(1 / r^{2}\right) \int_{E_{r}} w d a=0 .
$$

Proof. A result of Deny [1, p. 170] assures us that $a\left(E_{r}\right)$ is $o\left(r^{4}\right)$, and we can therefore take $w$ as the potential of a mass distribution $m$ on $\omega=N_{1 / 2}(0)$. Then

$$
\left(1 / r^{2}\right) \int_{E_{r}} w d a=-\int_{\omega}\left(1 / r^{2}\right) \int_{E_{r}} \log |z-\zeta| d a(z) d m(\zeta),
$$

so that it suffices to show that $\left(1 / r^{2}\right) \int_{E_{r}} \log |z-\zeta| d a(z)$ tends to zero uniformly for $\zeta$ on $\omega$. For any admissible value of $\zeta$ this integral does not decrease in magnitude when $E_{r}$ is replaced by a circular neighborhood $N_{R}(\zeta)$ of the same area. A direct calculation then yields

$$
\left|\left(1 / r^{2}\right) \int_{E_{r}} \log \right| z-\zeta|d a(z)| \leqq\left|\left(\pi R^{2} / r^{2}\right)(\log R-1 / 2)\right|,
$$

and the lemma follows.

An immediate consequence is

THEOREM 34. Let $w$ be an almost $\delta$-subharmonic function on an open subset $\Omega$ of $\mathcal{E}^{2}$. If $w$ admits a pseudo limit $A$ (finite or infinite) at $z \in \Omega$, then $\lim _{r \rightarrow 0} \alpha_{r} w(z)$ $=A$.

Since Lemma 3 is based on a result of Deny peculiar to the plane, it is to be expected that Theorem 34 does not extend in general to higher dimensional spaces. We have, in fact,

EXAmple 6. There exists a potential $w$ in $\varepsilon^{3}$ having pseudo limit 0 at the origin but for which the spatial mean over a sphere of radius $r$ about the origin tends to infinity as $r \rightarrow 0$. To construct this potential, we take $0<s<1$ and consider the sequence of points $\left(s^{k}, 0,0\right)$. For $k$ sufficiently large we can insert spheres $\sigma_{k}$ with centers on the $x$-axis and radii $r_{k}=s^{k} / k^{2}$ between the $k$ th and $(k+1)$ th points of the sequence. The Wiener criterion (Brelot [5]) shows that the set $E$ consisting of the union of these spheres is thin at the origin.

We define $w$ as the potential of the mass distribution formed by concentrating the mass $1 / k^{2}$ at the center of $\sigma_{k}$ and distributing the mass $-1 / k^{2}$ uniformly over the boundary of $\sigma_{k}$, for all admissible $k$. Since $w$ vanishes outside of $E$, it has pseudo limit 0 at the origin. However, a simple computation shows that the spatial mean tends to infinity.

Local properties allow a sharpening of some of the closure results of $\S 7$ for the case of quasi $\delta$-subharmonic functions.

REMARK 3. If we assume that the functions $w_{1}, w_{2}$, and $w$ in Theorems 
26,28 , and 29 are quasi $\delta$-subharmonic, then we can conclude that the functions $w_{1} w_{2}$ and $f \circ w$ are likewise quasi $\delta$-subharmonic.

This assertion follows trivially from the fact that two quasi $\delta$-subharmonic functions which are equal almost everywhere must actually be equal quasi everywhere (since each coincides quasi everywhere with its pseudo limit).

10. The characteristic function. In the theory of meromorphic functions the so-called "characteristic function" introduced by Nevanlinna [1] plays a central role. It is defined for an arbitrary meromorphic function $f$ by the formula

$T_{r}(f, z)=(1 / 2 \pi) \int_{0}^{2 \pi} \log ^{+}\left|f\left(z+r e^{i \theta}\right)\right| d \theta+\int_{0}^{r} \frac{p(t)-p(0)}{t} d t+p(0) \log r$,

where $p(t)$ denotes the number of poles of $f$ on the disc $S_{t}(z)$.

Privaloff [1] has given a direct extension of this definition to functions $w=u-v$, where $u$ and $v$ are subharmonic, by defining $T_{r}(w, z)$ according to the above formula, but with $w^{+}$replacing $\log ^{+}|f|$ and $p(t)$ denoting now the negative of the total mass of $v$ on $S_{t}(z){ }^{\left({ }^{19}\right)}$. Another extension of the Nevanlinna characteristic function has been given for potentials by Rosenbloom [1].

The Privaloff extension retains many of the properties of the original Nevanlinna characteristic function, such as those of being monotone nondecreasing in $r$ and convex in $\log r$. However, the situation is complicated somewhat by the fact that the improper integral $\int_{0}^{r}((p(t)-p(0)) / t) d t$ appearing in the Privaloff extension may fail to converge. That is, there may exist values of $z$ for which $T_{r}(w, z)$ does not exist finitely for any $r(>0)$. The derivation of Privaloff shows, in fact, that the finite existence of lim $\sup _{\zeta \rightarrow z}[v(\zeta)$ $-p(0) \log |z-\zeta|]$ is a necessary and sufficient condition for the finite existence of $T_{r}(w, z)$. This condition, which is certainly satisfied when the mass distribution consists of discrete point masses (as in the meromorphic case), does not hold in general $\left({ }^{20}\right)$.

We propose still another definition of the characteristic function.

Definition 8. Let $w$ be a function $\delta$-subharmonic on an open set $\Omega$, and let $(u, v)$ be a canonical representation of $w$. The upper envelope $\lambda$ of $u$ and $v$ will be termed a canonical envelope for $w$, and the function $T_{r}(w, z)$ defined for all circles $C_{r}(z)$ in $\Omega$ by the formula

$$
T_{r}(w, z)=\mu_{r} \lambda(z)
$$

will be called a characteristic function for $w$.

${ }^{(19)}$ Although $T_{r}(w, z)$ here depends on $v$, i.e. on the particular representation $(u, v)$ used in specifying $w$, it is clear that we have only to take $(u, v)$ as canonical to determine $T_{r}(w, z)$ uniquely.

(20) For example, the negative mass distribution obtained by distributing the mass $-1 / k^{2}$ uniformly on $C_{\theta}^{-k}(0)$, for every positive integer $k$, gives rise to a potential $v$ such that $\lim _{0} v$ $=-\infty$. 
This characteristic function is always finite and, by virtue of Theorem 5 , is given without reference to mass distributions. Furthermore, the point $z$ figuring in our definition need not even belong to $\Omega$, a fact which is essential in subsequent theorems. The exact relationship between the characteristic function defined here and those of Privaloff and Nevanlinna will be indicated later.

Since the canonical representations of $w$ are unique only to within the addition of a common harmonic function to each of the representing subharmonic functions, it follows that the characteristic functions are defined only to within an additive function of the form $\mu_{r} h(z)$, where $h$ is harmonic on $\Omega$. For this reason we introduce $\mathcal{T}_{\Omega}(w)$ to denote the equivalence class of all characteristic functions (considered as functions of $r$ and $z$ ) for the given $\delta$-subharmonic function $w$ and the given open set $\Omega\left({ }^{21}\right)$.

Certain elementary properties of the characteristic function are immediate.

THEOREM 35. Let $w, w_{1}$, and $w_{2}$ be functions $\delta$-subharmonic on an open set $\Omega$, and let $C_{r}(z)$ be any circle in $\Omega$. Then

(1) for $c$ any real number there exist characteristic functions such that

$$
T_{r}(c w, z)=|c| T_{r}(w, z) ;
$$

and

(2) for any choice of the indicated characteristic functions there exists a function $s$ subharmonic on $\Omega$ such that

$$
T_{r}\left(w_{1}+w_{2}, z\right) \leqq T_{r}\left(w_{1}, z\right)+T_{r}\left(w_{2}, z\right)-\mu_{r} s(z) .
$$

Proof. For (1) we take $(u, v)$ as a canonical representation of $w$ and observe that $|c| \max (u, v)$ is a canonical envelope for $c w$. To establish (2), we start with canonical representations $\left(u_{1}, v_{1}\right)$ and $\left(u_{2}, v_{2}\right)$ of $w_{1}$ and $w_{2}$, respectively, and let $(u, v)$ be a canonical representation of $w_{1}+w_{2}$. By Theorem 5 there exists a function $s$ subharmonic on $\Omega$ such that $u_{1}+u_{2}=u+s$ and $v_{1}+v_{2}=v+s$. Our assertion then follows from the evident inequality

$$
\max (u, v) \leqq \max \left(u_{1}, v_{1}\right)+\max \left(u_{2}, v_{2}\right)-s .
$$

An important special case of (1) is the symmetry condition $T_{r}(-w, z)$ $=T_{r}(w, z)$.

The following properties of characteristic functions are obvious from the subharmonicity of canonical envelopes.

TheOREM 36. Let $w$ be a function $\delta$-subharmonic on an open set $\Omega$ and

(21) Admittedly, the symbolism $T_{r}(w, z)$ involves an abuse of the functional notation' since the characteristic function depends not only on the open set $\Omega$ but also on the particular choice of canonical envelope $\lambda$. Use of a more cumbersome notation to avoid this inaccuracy does not seem warranted, and any ambiguity that might arise is easily sidestepped by inserting the proper quantifier. 
$A_{r_{1}, r_{2}}(z)$ an annulus contained in $\Omega$. If $T_{r}(w, z)$ is any characteristic function in $\mathcal{T}_{\Omega}(w)$, then $T_{r}(w, z)$ is convex in $\log r$ for $r$ on $\left(r_{1}, r_{2}\right)$.

THEOREM 37. Let $w$ be a function $\delta$-subharmonic on an open set $\Omega$ and $N_{R}(z)$ a neighborhood lying in $\Omega$. If $T_{r}(w, z)$ is any characteristic function in $\mathcal{T}_{\Omega}(w)$, then $T_{r}(w, z)$ is monotone nondecreasing in $r$ for $r$ on $(0, R)$.

THEOREM 38. Let $w$ be a function $\delta$-subharmonic on an open set $\Omega$, and let $\Omega_{r}$ be the set of all points $z$ for which $S_{r}(z) \subset \Omega$. Then, considered as a function of $z$ for $r$ fixed, any characteristic function $T_{r}(w, z)$ in $\mathcal{T}_{\Omega}(w)$ is a continuous subharmonic function on $\Omega_{r}$.

A theorem of Brelot [1, pp. 34-35] $\left.{ }^{22}\right)$ asserts that a function $u$ subharmonic on $N_{R}^{\prime}(0)=N_{R}(0)-(0)$ can be extended so as to be subharmonic at 0 if and only if $\lim _{r \rightarrow 0} \mu_{r} u^{+}(0)$ exists finitely. Moreover, Saks has shown (Radó $\left[1\right.$, p. 49]) that this condition can be replaced by $\lim _{r \rightarrow 0}\left[\mu_{r} u^{+}(0) / \log r\right]=0$. Since $u^{+}$is a canonical envelope for $u$, both of these results can be interpreted in terms of the characteristic function. It is natural, therefore, to look for $\delta$-subharmonic counterparts of theorems of this sort.

THEOREM 39. Let $w$ be a function $\delta$-subharmonic on a deleted neighborhood $N_{R}^{\prime}(0)$, and let $T_{r}(w, 0)$ be any characteristic function. Then a necessary and suffcient condition for $w$ to be $\delta$-subharmonic on the full neighborhood $N_{R}(0)$ is that $\lim _{r \rightarrow 0}\left[T_{r}(w, 0) / \log r\right]$ exist finitely.

Proof. The necessity of this condition results from the fact that the indicated limit equals the negative of the concentrated mass at 0 for the canonical envelope $\lambda$. For the sufficiency we employ a theorem of Brelot (Radó [1, 7.14]) which ensures the existence of a harmonic majorant $h$ for $\lambda$ on some deleted neighborhood of 0 . Obviously, $u-h$ and $v-h$ can be extended so as to be subharmonic at 0 , and the theorem follows.

Of course, $w$ may here fail to be defined, or to admit an extension of definition at 0 . However, if $w$ is defined, finitely or infinitely, at 0 , then $\lambda$ must have a finite value there. This leads to

CoROllary 39.1. Let w be a function $\delta$-subharmonic on $N_{R}^{\prime}(0)$. If $w$ admits an extension of definition at 0 so as to be $\delta$-subharmonic throughout $N_{R}(0)$, then there exists a characteristic function in $\mathcal{G}_{N^{\prime}{ }^{(0)}}(w)$ such that $\lim _{r \rightarrow 0}\left[T_{r}(w, 0) / \log r\right]$ $=0$.

Although the above condition is not sufficient for $w$ to admit an extension of definition at 0 preserving $\delta$-subharmonicity, we have by similar considerations

Corollary 39.2. A necessary and sufficient condition for a function w $\delta$-subharmonic on $N_{R}^{\prime}(0)$ to admit an extension of definition at 0 so as to be $\delta$-subharmonic on $N_{R}(0)$ is that there exist a characteristic function in $\mathcal{G}_{N^{\prime}{ }_{R}(0)}(w)$ such that $\lim _{r \rightarrow 0} T_{r}(w, 0)$ exists finitely.

${ }^{(22)}$ See also Radó [1, p. 49]. 
A further application of the characteristic function is concerned with the behavior of $w$ near the boundary, rather than in the neighborhood of a point.

THEOREM 40. A necessary and sufficient condition for a function $w \delta$-subharmonic on $N_{R}(0)$ to admit a representation $(u, v)$, where $u$ and $v$ are negative subharmonic functions, is that there exist a characteristic function $T_{r}(w, 0)$ bounded above for $r$ on $(0, R)$.

Proof. The necessity follows from Theorem 5 , since if $\left(u^{*}, v^{*}\right)$ is a canonical representation of $w$ there is a subharmonic function $s$ on $N_{R}(0)$ such that $u=u^{*}+s$ and $v=v^{*}+s$. Hence $T_{r}(w, 0) \leqq-\mu_{r} s(0)$, and the monotone nondecreasing nature of $\mu_{r} s(0)$ ensures that $T_{r}(w, 0)$ is bounded above. For the sufficiency we use the fact (Privaloff [2]) that $\mu_{r} \lambda(0)$ bounded above for $r$ on $(0, R)$ implies the existence of a harmonic majorant for the subharmonic function $\lambda$.

Invoking a theorem of Littlewood (Rado $[1,7.22]$ ) on the existence for almost all radial directions of the radial limit of a negative subharmonic function, we obtain

CoRollary 40.1. Let $w$ be a function $\delta$-subharmonic on $N_{R}(0)$. If there exists a characteristic function $T_{r}(w, 0)$ bounded above for $r$ on $(0, R)$, then $\lim _{r \rightarrow R-} w\left(r e^{i \theta}\right)$ exists finitely for almost all values of $\theta$.

Of significance in linking our characteristic function with those of Nevanlinna and Privaloff is the trivial observation that

$$
\max (u, v)=w^{+}+v
$$

holds whenever $w=u-v$. We apply this lattice identity to obtain alternative expressions for the characteristic function $T_{r}(w, z)$ considered as a function of $r$ for fixed $w$ and $z$.

THEOREM 41. Let $w$ be a function $\delta$-subharmonic on a region $\Omega \subset \varepsilon^{2}$, and let $p$ be the positive part of the mass distribution for w. Further, let $\Omega^{*}$ be a subregion of $\Omega$, and let $z$ be fixed such that $C_{r}(z)$ lies in $\Omega^{*}$ for all $r$ on $\left(r_{1}, r_{2}\right)$. If $p$ confined to $\Omega^{*}$ admits a potential, then for $T_{r}(w, z)$ any characteristic function there exist constants $A$ and $B$ such that

$$
\begin{aligned}
T_{r}(w, z)= & (1 / 2 \pi) \int_{0}^{2 \pi} w^{+}\left(z+r e^{i \theta}\right) d \theta \\
& +\int_{\Omega^{*}} \max (\log |z-\zeta|, \log r) d p(\zeta) \\
& +A \log r+B
\end{aligned}
$$

for $r$ on $\left(r_{1}, r_{2}\right)$.

Proof. From the formula 


$$
T_{r}(w, z)=\mu_{r} w^{+}(z)+\mu_{r} v(z)+\mu_{r} h(z),
$$

where $v$ is the potential of $-p$ confined to $\Omega^{*}$ and $h$ is a function harmonic on $\Omega^{*}$, we obtain the desired expression for $T_{r}(w, z)$ by evaluating $\mu_{r} v(z)$ and $\mu_{r} h(z)$.

A further specialization of the hypotheses yields

CoROllaRY 41.1. Let w be a function $\delta$-subharmonic on $N_{R}^{\prime}(z)$, p the positive part of the mass distribution for $w$, and $P(t)$ the mass $p\left[S_{t}(z)\right]$. If $p$ admits a potential finite at $z$, then for $T_{r}(w, z)$ any characteristic function there exist constants $A$ and $B$ such that

$$
\begin{aligned}
T_{r}(w, z)= & (1 / 2 \pi) \int_{0}^{2 \pi} w^{+}\left(z+r e^{i \theta}\right) d \theta \\
& +\int_{N^{\prime}{ }_{r}(z)} \log \frac{r}{|z-\zeta|} d p(\zeta)+A \log r+B
\end{aligned}
$$

$$
\begin{aligned}
& =(1 / 2 \pi) \int_{0}^{2 \pi} w^{+}\left(z+r e^{i \theta}\right) d \theta \\
& +\int_{0}^{r} \frac{P(t)-P(0)}{t} d t+A \log r+B
\end{aligned}
$$

for $r$ on $(0, R)$. If further $w$ is $\delta$-subharmonic at $z$, and $T_{r}(w, z) \in \mathcal{T}_{N_{R}(z)}(w)$, then $A=P(0)$.

Proof. Given $r \in(0, R)$, we fix $\rho \in(r, R)$ and denote by $v_{\rho}$ the potential of - $p$ confined to $N_{\rho}^{\prime}(z)$. Since $v_{\rho}$ is finite at $z$, formula (10.2) remains in force when we subtract the constant $v_{\rho}(z)$ from its right-hand member. Formulas (10.3) then follow from (10.2) and the equalities

$$
\begin{aligned}
\int_{N^{\prime}{ }_{\rho}(z)} \max (\log |z-\zeta|, \log r) d p(\zeta)-v_{\rho}(z) & \\
=\int_{N^{\prime}(z)} \log \frac{r}{|z-\zeta|} d p(\zeta) & =\int_{0}^{r} \frac{P(t)-P(0)}{t} d t .
\end{aligned}
$$

That $A=P(0)$ when $w$ is $\delta$-subharmonic at $z$ is readily seen from the fact that the function $h$ in the proof of Theorem 41 must then be the potential of the concentrated mass at $z$ plus a function harmonic on $N_{\rho}(z)$.

A slightly different expression for the characteristic function can be derived by methods paralleling those given above if we take cognizance of the relation

$$
2 \max (u, v)=|w|+u+v,
$$

where $w=u-v$. We thus arrive at the following theorem and corollary, appli- 
cations of which will be made in a subsequent paper on entire $\delta$-subharmonic functions and functions of potential type.

THEOREM 42. Let $w$ be a function $\delta$-subharmonic on a region $\Omega \subset \mathcal{E}^{2}$, and let $m$ be the mass distribution for $w$. Further, let $\Omega^{*}$ be a subregion of $\Omega$, and let $z$ be fixed such that $C_{r}(z)$ lies in $\Omega^{*}$ for all $r$ on $\left(r_{1}, r_{2}\right)$. If $m$ confined to $\Omega^{*}$ admits a potential, then for $T_{r}(w, z)$ any characteristic function there exist constants $A$ and $B$ such that

$$
\begin{aligned}
T_{r}(w, z)= & (1 / 4 \pi) \int_{0}^{2 \pi}\left|w\left(z+r e^{i \theta}\right)\right| d \theta \\
& +(1 / 2) \int_{\Omega^{*}} \max (\log |z-\zeta|, \log r)|d m(\zeta)|+A \log r+B
\end{aligned}
$$

for $r$ on $\left(r_{1}, r_{2}\right)$.

Corollary 42.1. Let $w$ be a function $\delta$-subharmonic on $N_{R}^{\prime}(z), m$ the mass distribution for $w$, and $\Phi(t)$ the total variation of $m$ on $S_{t}(z)$. If $m$ admits a potential finite at $z$, then for $T_{r}(w, z)$ any characteristic function there exist constants $A$ and $B$ such that

$$
\begin{aligned}
T_{r}(w, z)= & (1 / 4 \pi) \int_{0}^{2 \pi}\left|w\left(z+r e^{i \theta}\right)\right| d \theta \\
& +(1 / 2) \int_{N^{\prime} r(z)} \log \frac{r}{|z-\zeta|}|d m(\zeta)|+A \log r+B \\
= & (1 / 4 \pi) \int_{0}^{2 \pi}\left|w\left(z+r e^{i \theta}\right)\right| d \theta \\
& +(1 / 2) \int_{0}^{r} \frac{\Phi(t)-\Phi(0)}{t} d t+A \log r+B
\end{aligned}
$$

for $r$ on $(0, R)$. If further $w$ is $\delta$-subharmonic at $z$ and $T_{r}(w, z) \in \mathcal{G}_{N_{R}(z)}(w)$, then $A=\Phi(0)$.

The correlation between our characteristic function and that of Privaloff is at once evident from Corollary 41.1: at those points for which the Privaloff characteristic function is defined finitely the two characteristic functions, considered as functions of $r$, differ only by a constant. This observation of course applies also to the Nevanlinna characteristic function, since the latter appears as a special case of the Privaloff characteristic function.

We remark in passing that it is possible to formulate a characteristic function along the lines of Definition 7 but with an areal mean replacing the circumferential mean. Moreover, we get still another characteristic function by setting $T_{r}(w, z)=\max \left[\mu_{r} u(z), \mu_{r} v(z)\right]$. Many of our basic results re- 
main valid for these two variants of the characteristic function, but equation (10.3), bridging the gap with the classical theory, is no longer available.

\section{Notational GLOSSARY}

$\mathcal{E}^{n}=n$-dimensional Euclidean space.

$C_{r}(z)=\{\zeta:|\zeta-z|=r\}$.

$N_{r}(z)=\{\zeta:|\zeta-z|<r\}$.

$S_{r}(z)=\{\zeta:|\zeta-z| \leqq r\}$.

$N_{r}^{\prime}(z)=N_{r}(z)-(z)$.

$\mu_{r} w(z)=(1 / 2 \pi) \int_{0}^{2 \pi} w\left(z+r e^{i \theta}\right) d \theta$.

$\alpha_{r} w(z)=\left(1 / \pi r^{2}\right) \int_{0}^{r} \int_{0}^{2 \pi} w\left(z+\rho e^{i \theta}\right) \rho d \theta d \rho$.

$\partial E=$ boundary of $E$.

$a(E)=2$-dimensional Lebesgue measure of $E$.

$\phi_{E}(w)=$ total variation on $E$ of the mass distribution for $w$.

\section{BIBLIOGRAPHY}

C. R. Adams and J. A. Clarkson

1. On definitions of bounded variation for functions of two variables, Trans. Amer. Math. Soc. vol. 35 (1933) pp. 824-854.

2. Properties of functions $f(x, y)$ of bounded variation, Trans. Amer. Math. Soc. vol. 36 (1934) pp. 711-730.

\section{S. BANACH}

1. Théorie des opérations linéaires, Warsaw, 1932.

\section{BRELOT}

1. Etude des fonctions sousharmoniques au voisinage d'un point, Actualités Scientifiques et Industrielles, vol. 139, 1934, pp. 5-55.

2. Sur l'allure des fonctions harmoniques et sousharmoniques à la frontière, Mathematische Nachrichten vol. 4 (1950) pp. 248-307.

3. Sur l'intégration de $\Delta u(M)=\phi(M)$, C. R. Acad. Sci. Paris vol. 201 (1945) pp. 1316-1318.

4. Minorantes sousharmoniques, extrémales et capacité, J. de Math. vol. 24 (1945) pp. 1-32.

5. Sur les ensembles effilés, Bull. Sci. Math. vol. 68 (1944) pp. 12-36.

6. Deux théorèmes généraux sur le potentiel et quelgues applications, C. R. Acad. Sci. Paris, vol. 226 (1948) pp. 1499-1500.

\section{H. CARTAN}

1. Théorie du potentiel Newtonien: énergie, capacité, suites de potentiels, Bull. Soc. Math. France vol. 73 (1945) pp. 74-106.

J. DENY

1. Sur les infinis d'un potentiel, C. R. Acad. Sci. Paris vol. 224 (1947) pp. 524-525.

2. Les potentiels d'energie finie, Acta Math. vol. 82 (1950) pp. 107-183.

\section{G. C. Evans}

1. Complements of potential theory, part II, Amer. J. Math. vol. 55 (1933) pp. 29-49.

E. HewITT

1. Remarks on the inversion of Fourier-Stieltjes transforms, Ann. of Math. vol. 57 (1953) pp. 458-474.

R. NeVANLINNA

1. Eindeutige analytische Funktionen, Berlin, 1936.

E. E. Privaloff

1. A generalization of Jensen's formula, part I, Izvestia Akad. Nauk. vol. 6-7 (1935) pp. 837-847. 
2. Subharmonic functions, Moscow, 1937.

T. RADó

1. Subharmonic functions, Berlin, 1937.

F. RIESZ

1. Sur certains systèmes singuliers d'équations intégrales, Ann. Ecole Norm. vol. 28 (1911) pp. 5-62.

P. C. Rosenbloom

1. Mass distributions and their potentials, Proceedings of the 11th Scandinavian Mathematical Congress, Trondheim, Norway, 1949, pp. 130-138.

W. RUDIN

1. Integral representation of continous functions, Trans. Amer. Math. Soc. vol. 68 (1950) pp. 278-286.

S. SAKS

1. Theory of the integral, 2d rev. ed., Warsaw-Lwów, 1937.

L. SCHWARTZ

1. Théorie des distributions, vol. I, Actualités Scientifiques et Industrielles, no. 1091, 1950.

2. Théorie des distributions, vol. II, Actualités Scientifiques et Industrielles, no. 1122, 1951.

J. L. WALSH

1. The approximation of harmonic functions by harmonic polynomials and by harmonic rational functions, Bull. Amer. Math. Soc. vol. 35 (1929) pp. 499-544.

N. WIENER

1. Laplacians and continuous linear functionals, Acta Szeged vol. 3 (1927) pp. 7-16.

S. ZAREMBA

1. Contribution d la théorie d'une équation fonctionelle de la physique, Rend. Circ. Mat. Palermo vol. 19 (1905) pp. 140-150.

BROWN UNIVERSITY,

Providence, R. I.

UNIVERSITY OF WASHINGTON,

SEATtLE, Wash. 